TITLE:

\title{
Decay of a Linear Pendulum in a Free-Molecular Gas and in a Special Lorentz Gas
}

\author{
$\operatorname{AUTHOR}(\mathrm{S}):$ \\ Tsuji, Tetsuro; Aoki, Kazuo
}

CITATION:

Tsuji, Tetsuro ... [et al]. Decay of a Linear Pendulum in a Free-Molecular Gas and in a Special Lorentz Gas. Journal of Statistical Physics 2012, 146(3): 620-645

ISSUE DATE:

2012-02

URL:

http://hdl.handle.net/2433/153297

\section{RIGHT:}

The final publication is available at www.springerlink.com; この論文は 出版社版でありません。引用の際には出版社版をご確認ご利用くださ $\omega_{\circ}$; This is not the published version. Please cite only the published version. 
manuscript No.

(will be inserted by the editor)

Tetsuro Tsuji · Kazuo Aoki

\title{
Decay of a linear pendulum in a free- molecular gas and in a special Lorentz gas
}

Received: date / Accepted: date

\begin{abstract}
A circular disk without thickness is placed in a gas, and an external force, obeying Hooke's law, is acting perpendicularly on the disk. If the disk is displaced perpendicularly from its equilibrium position and released, then it starts an oscillatory or non-oscillatory unsteady motion, which decays as time goes on because of the drag exerted by the gas molecules. This unsteady motion, i.e., the decay of this linear pendulum, is investigated numerically, under the diffuse reflection condition on the surface of the disk, with special interest in the manner of its decay, for two kinds of gases: one is a collisionless gas (or Knudsen gas) and the other is a special Lorentz gas interacting with a background. It is shown that the decay of the displacement of the disk is slow and is in proportion to an inverse power of time for the collisionless gas. The result complements the existing mathematical study of a similar problem [S. Caprino, et al., Math. Models. Meth. Appl. Sci. 17, 1369-1403 (2007)] in the case of non-oscillatory decay. It is also shown that the manner of the decay changes significantly for the special Lorentz gas.
\end{abstract}

Keywords Decay of pendulum $\cdot$ Free-molecular gas $\cdot$ Lorentz gas $\cdot$ Kinetic theory of gases

PACS 05.20.Dd * 47.45.Dt · 51.10.+y · 47.45.Ab · 47.45.-n

This work was supported by the grant-in-aid for scientific research No. 23360048 from JSPS.

T. Tsuji

Department of Mechanical Engineering and Science

Kyoto University, Kyoto 606-8501, Japan

E-mail: t.tsuji@ft2.ecs.kyoto-u.ac.jp

K. Aoki

Department of Mechanical Engineering and Science

and Advanced Research Institute of Fluid Science and Engineering

Kyoto University, Kyoto 606-8501, Japan

E-mail: aoki.kazuo.7a@kyoto-u.ac.jp 


\section{Introduction}

Let us consider a body in an infinite expanse of a gas. The body is supposed to be subject to an external force that obeys Hooke's law (i.e., a restoring force in proportion to the displacement from the equilibrium position) and be movable only in the direction parallel to the force (linear pendulum). If the body is displaced and released with an initial velocity, then it starts an unsteady motion, e.g., an oscillation around the equilibrium position, but the motion attenuates as time goes on because of the drag force exerted on the body by the surrounding gas. We are concerned with the rate of decay of the motion of the body.

If the drag force is proportional to the speed of the body, the motion of the body (e.g., the amplitude of the oscillation) decays exponentially in time. However, the drag exerted by the gas is not so simple, and we may expect a different decay rate. This problem, together with the case in which the body is subject to a constant external force and approaches a final steady motion, has been studied extensively [1-6] when the surrounding gas is a collisionless gas (a free-molecular gas or the Knudsen gas), i.e., a gas that is so rarefied that collisions between gas molecules can be neglected.

For instance, the case where the body is a circular disk and the external force, obeying Hooke's law, acts perpendicularly on the disk was studied mathematically in [2]. To be more specific, let us denote by $t_{*}$ the time variable and by $X_{w}\left(t_{*}\right)$ the displacement (with sign) of the disk from the equilibrium position. Then, the force is expressed as $-\kappa X_{w}\left(t_{*}\right)$ with a positive constant $\kappa$. The gas molecules are assumed to undergo specular reflection on the disk. Initially, the disk is fixed with displacement $X_{w 0}$, and the gas is in a uniform equilibrium state at rest. At time $t_{*}=0$, it is released with an initial velocity (parallel to the external force). Then, the disk starts an unsteady motion, and as time goes on, the motion decays, i.e., $X_{w}\left(t_{*}\right) \rightarrow 0$. In [2], it is proved that there exist cases where $X_{w}\left(t_{*}\right)$ decays monotonically (without oscillation) and that the decay is slow and algebraic in such cases, i.e., it is described as

$$
\left|X_{w}\left(t_{*}\right)\right| \approx C_{s} / t_{*}^{d+2},
$$

for sufficiently large $t_{*}$, where $d$ is the dimension of the problem and $C_{s}$ is a positive constant. A subsequent numerical study [6] confirmed an algebraic decay even in the case with many oscillations for the one-dimensional problem $(d=1)$. Here, it should be mentioned that, since the diffuse-reflection condition, rather than the specular-reflection condition, was employed in [6], the decay rate was different and was proportional to $1 / t_{*}^{2}$, which is slower than Eq. (1) with $d=1$. These algebraically slow decays are attributed to a long-memory effect peculiar to a collisionless gas. In such a gas, the molecules that are reflected by the disk at early times may hit the disk again at later times. In contrast to a gas with intermolecular collisions, such molecules transfer information about the disk at an early stage directly to the disk at a later stage and may affect the motion of the disk long time later. In other words, they give rise to a long-memory effect.

The aim of the present study is twofold. One is to extend the numerical study in [6] to two- and three-dimensional problems and provide numerical evidence of the decay rate described by

$$
\left|X_{w}\left(t_{*}\right)\right| \approx C_{f} / t_{*}^{d+1},
$$


with a positive constant $C_{f}$, under the diffuse-reflection boundary condition. The other is to see the change of the decay rate when the long-memory effect is destroyed by introducing a sort of interaction of gas molecules with a background. For this purpose, we consider a special Lorentz gas explained in the main text and consider the resulting kinetic equation (a toy model). We study the decay of the unsteady motion of the disk numerically and show that the decay rate changes significantly.

\section{Formulation of the Problem}

\subsection{Problem}

Let us consider an infinite expanse of a rarefied gas in an equilibrium state at rest at temperature $T_{* 0}$ and density $\rho_{* 0}$. What kind of gas (i.e., what kind of kinetic equation) we are considering will be specified in Sec. 2.2. A circular disk with diameter $L_{*}$ and without thickness, kept at temperature $T_{* 0}$, is placed in the gas, and an external force, obeying Hooke's law (elastic force), is acting on the disk perpendicularly to its surface. In the present study, we consider $d$-dimensional problems with $d=1,2$, and 3: The disk is a circular disk with diameter $L_{*}$ when $d=3$ [Fig. 1(a)], an infinite plate with finite width $L_{*}$ when $d=2$ [Fig. 1(b)], and an infinitely wide plate when $d=1$ [Fig. 1(c)]. Let $t_{*}$ be the time variable and $X_{i}$ be the Cartesian coordinates in space with the $X_{i}$ axes taken as in Fig. 1. That is, $X_{1}$ axis is taken parallel to the elastic force, and $X_{1}=0$ indicates the equilibrium position of the disk. Therefore, the elastic force $F_{1}$ per unit area of the disk is expressed as

$$
F_{1}=-M_{*} \omega_{*}^{2} X_{w}\left(t_{*}\right),
$$

where $X_{w}\left(t_{*}\right)$ is the position ( $X_{1}$ coordinate) of the disk at time $t_{*}, M_{*}$ is the mass density of the disk (the mass of the disk per unit surface), and $\omega_{*}$ is the proper frequency of the elastic force.

At time $t_{*}=0$, the disk is released from a position $X_{1}=X_{w 0}$ with an initial velocity $V_{w 0}$ in the $X_{1}$ direction. Then, it starts an unsteady motion (e.g., an oscillatory motion), but the motion decays as time goes on because of the drag exerted on the disk by the surrounding gas. We investigate this unsteady motion of the disk numerically, with special interest in the long-time behavior and the manner of the decay, assuming that the disk moves only along the $X_{1}$ axis and does not change its attitude.

\subsection{Choice of the gas}

In the present study, we consider the following two types of gas as the gas surrounding the disk. We denote by $\xi_{i}$ the molecular velocity and by $f_{*}\left(X_{i}, \xi_{i}, t_{*}\right)$ the velocity distribution function of the gas molecules.

\subsubsection{Collisionless gas}

The collisionless gas is a gas that is so rarefied that collisions between gas molecules can be neglected. It is also called the free-molecular gas or the Knudsen gas. In 




(a)



(b)

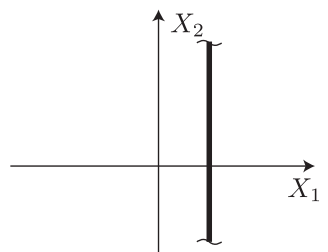

(c)

Fig. 1 A circular disk of $d$ dimension $(d=1,2,3)$ without thickness in a gas. (a) Circular disk with diameter $L_{*}(d=3)$, (b) infinite plate with finite width $L_{*}(d=2)$, and (c) infinite plate $(d=1)$.

this case, the basic equation is the Boltzmann equation without the collision term, i.e., the free-transport equation:

$$
\frac{\partial f_{*}}{\partial t_{*}}+\xi_{j} \frac{\partial f_{*}}{\partial X_{j}}=0
$$

That is, $f_{*}$ is constant along the molecular trajectory.

\subsubsection{A kind of the Lorentz gas}

In addition to the collisionless gas, we consider a gas that is described by the following equation.

$$
\frac{\partial f_{*}}{\partial t_{*}}+\xi_{i} \frac{\partial f_{*}}{\partial X_{i}}=\nu\left(\xi_{i}\right)\left(f_{* \infty}-f_{*}\right),
$$

where

$$
\begin{aligned}
\nu\left(\xi_{i}\right) & =\frac{\pi\left(r+r_{s}\right)^{2}}{m_{s}} \int_{\mathbb{R}^{3}}\left|\boldsymbol{\xi}-\boldsymbol{\xi}_{s}\right| f_{* s}\left(\xi_{s i}\right) d \boldsymbol{\xi}_{s}, \\
f_{* \infty}\left(\xi_{i}\right) & =\frac{\pi\left(r+r_{s}\right)^{2}}{m_{s} \nu\left(\xi_{i}\right)} \int_{\mathbb{R}^{3}}\left|\boldsymbol{\xi}-\boldsymbol{\xi}_{s}\right| f_{* s}\left(\xi_{s i}\right) f_{* v}\left(\xi_{i}-\xi_{s i}\right) d \boldsymbol{\xi}_{s},
\end{aligned}
$$

with $d \boldsymbol{\xi}_{s}=d \xi_{s 1} d \xi_{s 2} d \xi_{s 3}$. Here, $f_{* v}\left(\xi_{i}\right)$ and $f_{* s}\left(\xi_{i}\right)$ are given functions of $\xi_{i}$, and $r, r_{s}$, and $m_{s}$ are given constants. A physical model that leads to this equation is the following.

Let us suppose that the gas is a vapor of a substance and its molecule is a hard sphere with mass $m$ and radius $r$. In the gas, there are randomly distributed spherical obstacles (droplets) of mass $m_{s}$ and radius $r_{s}$, which are made of the condensed phase of the same substance as the vapor. The obstacles are moving with velocity $\xi_{s i}$, and their velocity is distributed according to a given distribution $f_{* s}\left(\xi_{s i}\right)$ that is uniform in $X_{i}$ and constant in $t_{*}$. We assume that the vapor molecules hitting an obstacle are absorbed and reemitted with a velocity distribution $f_{* v}\left(\xi_{i}-\xi_{s i}\right)$, which is a given function of $\xi_{i}-\xi_{s i}$, uniform in $X_{i}$, and constant in $t_{*}$. We neglect the collisions between vapor molecules and consider their interaction with the obstacles only. Then, we arrive at Eq. (5). See Appendix for more details. 
In the actual analysis, we use an equation that is a further simplification of Eq. (5), derived under the assumption that $f_{* v}$ and $f_{* s}$ are Maxwellians and the average speed of motion of the obstacles are much smaller than that of the gas molecules (see Appendix).

\subsection{Assumptions}

We investigate the problem stated in Sec. 2.1 under the following assumptions:

(i) The behavior of the gas is described by Eq. (4) or the model equation Eq. (12) shown later, which is a simplified version of Eq. (5).

(ii) The gas molecules undergo diffuse reflection on the surface of the disk. That is. the molecules reflected by the disk are distributed according to the Maxwellian distribution characterized by the temperature and velocity of the disk, and there is no net mass flux across the disk surface.

In the two-dimensional (2D) problem $(d=2), f_{*}$ is independent of $X_{3}$; in the one-dimensional (1D) problem $(d=1), f_{*}$ is independent of $X_{2}$ and $X_{3}$.

\section{Basic Equations}

In this section, we summarize the basic equations in dimensionless form. We restrict ourselves to the three-dimensional problem, since the two- and one-dimensional cases are essentially the same.

\subsection{Dimensionless variables and notations}

In the preceding sections, we have introduced dimensional variables $t_{*}, X_{i}, \xi_{i}, f_{*}$, $X_{w}$, and $V_{w}$ and dimensional quantities $T_{* 0}, \rho_{* 0}, L_{*}, M_{*}, \omega_{*}, X_{w 0}$, and $V_{w 0}$. In addition, let us denote by $G_{*}\left[f_{*}\right]$ the drag acting on the disk divided by its area and by $f_{* 0}$ the Maxwellian distribution at rest at density $\rho_{* 0}$ and temperature $T_{* 0}$.

Then we introduce the dimensionless quantities $t, x_{i}, \zeta_{i}, f, x_{w}, v_{w}, L, M$, $x_{w 0}, v_{w 0}, G[f]$, and $f_{0}$ by the following relations:

$$
\begin{aligned}
& t_{*}=\left(1 / \omega_{*}\right) t, \quad X_{i}=\left(\sqrt{2 R_{*} T_{* 0}} / \omega_{*}\right) x_{i}, \quad \xi_{i}=\sqrt{2 R_{*} T_{* 0}} \zeta_{i}, \\
& f_{*}\left(X_{i}, \xi_{i}, t_{*}\right)=\left[\rho_{* 0} /\left(2 R_{*} T_{* 0}\right)^{3 / 2}\right] f\left(x_{i}, \zeta_{i}, t\right), \\
& X_{w}\left(t_{*}\right)=\left(\sqrt{2 R_{*} T_{* 0}} / \omega_{*}\right) x_{w}(t), \quad V_{w}\left(t_{*}\right)=\sqrt{2 R_{*} T_{* 0}} v_{w}(t), \\
& L_{*}=\left(\sqrt{2 R_{*} T_{* 0}} / \omega_{*}\right) L, \quad M_{*}=\left(\rho_{* 0} \sqrt{2 R_{*} T_{* 0}} / \omega_{*}\right) M, \\
& X_{w 0}=\left(\sqrt{2 R_{*} T_{* 0}} / \omega_{*}\right) x_{w 0}, \quad V_{w 0}=\sqrt{2 R_{*} T_{* 0}} v_{w 0}, \\
& G_{*}\left[f_{*}\right]=\rho_{0 *}\left(2 R_{*} T_{* 0}\right) G[f], \\
& f_{* 0}(|\boldsymbol{\xi}|)=\left[\rho_{* 0} /\left(2 R_{*} T_{* 0}\right)^{3 / 2}\right] f_{0}(|\boldsymbol{\zeta}|),
\end{aligned}
$$


where $R_{*}$ is the gas constant per unit mass $\left(R_{*}=k_{B} / m\right.$ with $k_{B}$ the Boltzmann constant and $m$ the mass of a gas molecule), and thus $f_{0}$ is given by

$$
f_{0}(|\boldsymbol{\zeta}|)=\pi^{-3 / 2} \exp \left(-|\boldsymbol{\zeta}|^{2}\right) .
$$

We further introduce some additional notations for later convenience: $c_{1}$ is the molecular velocity relative to the velocity of the disk in the $x_{1}$ direction, i.e.,

$$
c_{1}=\zeta_{1}-v_{w}(t)
$$

$\partial S_{ \pm}(t)$ is the surface of the disk, i.e.,

$$
\partial S_{ \pm}(t)=\left\{x_{i} \in \mathbb{R}^{3} \mid x_{1}=x_{w}(t) \pm 0, x_{2}^{2}+x_{3}^{2}<L^{2} / 4\right\},
$$

and $\Omega_{ \pm}(t)$ is the range of molecular velocity defined by

$$
\Omega_{ \pm}(t)=\left\{\zeta_{i} \in \mathbb{R}^{3} \mid c_{1} \gtrless 0\right\} .
$$

In Eqs. (10) and (11) and in what follows, the upper (or lower) signs go together.

\subsection{Basic equations in dimensionless form}

Our basic equation is Eq. (82) in Appendix, which is a toy model and is a simplified version of Eq. (5), i.e.,

$$
\frac{\partial f}{\partial t}+\zeta_{i} \frac{\partial f}{\partial x_{i}}=\frac{\nu_{\epsilon}(|\zeta|)}{\mathrm{Kn}}\left(f_{0}-f\right)
$$

The case of a collisionless gas [Eq. (4)] is included in this equation as a special case $(\mathrm{Kn} \rightarrow \infty)$. In Eq. (12), the dimensionless collision frequency $\nu_{\epsilon}(|\zeta|)$ is defined by the following function $\nu_{\epsilon}(x)$ :

$$
\nu_{\epsilon}(x)=\frac{\epsilon}{\sqrt{\pi}}\left[\exp \left(-\frac{x^{2}}{\epsilon^{2}}\right)+\sqrt{\pi}\left(\frac{x}{\epsilon}+\frac{\epsilon}{2 x}\right) \operatorname{erf}\left(\frac{x}{\epsilon}\right)\right], \quad \text { for } \quad x>0,
$$

with $\operatorname{erf}(x)$ the error function given by

$$
\operatorname{erf}(x)=\frac{2}{\sqrt{\pi}} \int_{0}^{x} \exp \left(-y^{2}\right) d y,
$$

and the Knudsen number $\mathrm{Kn}$ is defined as

$$
\mathrm{Kn}=\left[\pi n_{* s}\left(r+r_{s}\right)^{2}\right]^{-1}\left(\sqrt{2 R_{*} T_{* 0}} / \omega_{*}\right)^{-1},
$$

with $n_{* s}$ a positive constant [the number density of the obstacles; cf. Eq. (62)]. Here, $\epsilon$ is a small parameter, and the $\mathrm{Kn}$ is the ratio between the mean free path of a gas molecule for the collisions against obstacles [see Eqs. (81) and (82c)] and the characteristic length $\left(\sqrt{2 R_{*} T_{* 0}} / \omega_{*}\right)$. See Appendix for the details of the derivation of Eq. (12) and the physical meaning of the parameters and conditions.

The corresponding initial condition is given by

$$
f\left(x_{i}, \zeta_{i}, 0\right)=f_{0}\left(\zeta_{i}\right)
$$


and the boundary condition (diffuse reflection) on the surface of the disk $\partial S_{ \pm}(t)$ is given by

$$
f\left(x_{i}, \zeta_{i}, t\right)=f_{w \pm}\left(x_{2}, x_{3}, \zeta_{i}, t\right), \quad \text { for } \quad x_{i} \in \partial S_{ \pm}(t), \quad \zeta_{i} \in \Omega_{ \pm}(t) .
$$

where

$$
\begin{aligned}
& f_{w \pm}\left(x_{2}, x_{3}, \zeta_{i}, t\right)=\pi^{-3 / 2} \rho_{w \pm}\left(x_{2}, x_{3}, t\right) \exp \left(-c_{1}^{2}-\zeta_{2}^{2}-\zeta_{3}^{2}\right), \\
& \rho_{w \pm}\left(x_{2}, x_{3}, t\right)=\mp 2 \sqrt{\pi} \int_{\Omega_{\mp}(t)} c_{1} f\left(x_{i}, \zeta_{i}, t\right) d \boldsymbol{\zeta}, \quad \text { for } x_{i} \in \partial S_{ \pm}(t),
\end{aligned}
$$

with the notation $d \boldsymbol{\zeta}=d \zeta_{1} d \zeta_{2} d \zeta_{3}$.

The equation of motion of the disk is given as

$$
\frac{d x_{w}(t)}{d t}=v_{w}(t), \quad \frac{d v_{w}}{d t}=-x_{w}-\frac{1}{M} G[f]
$$

with the initial condition

$$
x_{w}(0)=x_{w 0}, \quad v_{w}(0)=v_{w 0} .
$$

The dimensionless average drag $G[f]$ per unit area is expressed in terms of the velocity distribution function on the disk:

$$
G[f]=G_{+}[f]+G_{-}[f],
$$

where

$$
\begin{aligned}
G_{ \pm}[f]= \pm \frac{1}{\pi(L / 2)^{2}} \int_{x_{2}^{2}+x_{3}^{2}<L^{2} / 4}\left(\int_{\Omega_{\mp}(t)} c_{1}^{2} f\left(x_{w}(t) \pm 0, x_{2}, x_{3}, \zeta_{i}, t\right) d \boldsymbol{\zeta}\right. \\
\left.+\int_{\Omega_{ \pm}(t)} c_{1}^{2} f_{w \pm}\left(x_{2}, x_{3}, \zeta_{i}, t\right) d \boldsymbol{\zeta}\right) d x_{2} d x_{3}
\end{aligned}
$$

We solve the coupled system Eqs. (12)-(18) and Eqs. (19)-(22) numerically with special interest in the manner of decay of the motion of the disk, i.e., the decay of $\left|x_{w}\right|$. The parameters contained in the present problem are

$$
L, \quad M, \quad x_{w 0}, \quad v_{w 0}, \quad \mathrm{Kn}, \quad \epsilon
$$

Recall that $\mathrm{Kn}=\infty$ recovers the collisionless gas Eq. (4). 


\section{Preliminaries}

\subsection{Integral form}

In the numerical analysis, we solve Eqs. (12)-(18) and Eqs. (19)-(22) in a timemarching manner. For this purpose, it is more convenient to use the expression of $\rho_{w \pm}\left(x_{2}, x_{2}, t\right)$ in terms of the integrals containing information about $\rho_{w \pm}, x_{w}$, and $v_{w}$ in the past. In order to derive the integral form of $\rho_{w \pm}$, let us fix the time $t$ and assume that $\rho_{w \pm}, x_{w}$, and $v_{w}$ are known for the time $s$ in the past $(0 \leq s<t)$. In addition, we consider $x_{i}$ on the disk $\left[x_{i} \in \partial S_{ \pm}(t)\right]$.

For each velocity $\zeta_{i}$ for the molecules incident on the disk $\left[\zeta_{i} \in \Omega_{\mp}(t)\right.$ for $x_{i} \in \partial S_{ \pm}(t)$ ], we trace back the corresponding molecular trajectory. Then, we either (i) reach the initial time without hitting the disk, or (ii) encounter the disk at a time $t^{b}$ in the past $\left(0<t^{b}<t\right)$ at a point $\left(x_{w}\left(t^{b}\right) \pm 0, x_{2}^{b}, x_{3}^{b}\right)$ on the disk. To be more specific, $t^{b}\left(\zeta_{1}, t\right)$ is given by

$$
t^{b}=\max \left\{s \in(0, t) \mid x_{1}-\zeta_{1}(t-s)=x_{w}(s)\right\},
$$

and $x_{2}^{b}\left(x_{2}, \zeta_{2}, t, t^{b}\right)$ and $x_{3}^{b}\left(x_{3}, \zeta_{3}, t, t^{b}\right)$ by

$$
x_{2}^{b}=x_{2}-\zeta_{2}\left(t-t^{b}\right), \quad x_{3}^{b}=x_{3}-\zeta_{3}\left(t-t^{b}\right),
$$

under the condition that $\left(x_{2}^{b}\right)^{2}+\left(x_{3}^{b}\right)^{2}<(L / 2)^{2}$. The latter condition restricts the range of $\zeta_{i}$ and is stated more precisely that

$$
\begin{aligned}
& \zeta_{i} \in \Gamma_{ \pm}\left(x_{2}, x_{3}, t\right), \\
& \Gamma_{ \pm}=\left\{\zeta_{i} \in \mathbb{R}^{3} \mid{ }^{\exists} t^{b}>0,\left(x_{2}^{b}\right)^{2}+\left(x_{3}^{b}\right)^{2}<(L / 2)^{2}, x_{i} \in \partial S_{ \pm}(t)\right\} .
\end{aligned}
$$

Now let us define $\tau_{ \pm}^{b}\left(x_{i}, \zeta_{i}, t\right)$ by

$$
\tau_{ \pm}^{b}= \begin{cases}t^{b}, & \text { for } \quad \zeta_{i} \in \Gamma_{ \pm} \\ 0, & \text { for } \quad \zeta_{i} \notin \Gamma_{ \pm} .\end{cases}
$$

This quantity is similar to the backward exit time for free-transport particles in a closed domain. Then, integrating Eq. (12) along the characteristic line, one can write

$$
\begin{aligned}
f\left(x_{i}, \zeta_{i}, t\right)=f_{0}+\left[f \left(x_{i}-\zeta_{i}(\right.\right. & \left.\left.\left.t-\tau_{ \pm}^{b}\right), \zeta_{i}, \tau_{ \pm}^{b}\right)-f_{0}\right] \\
& \times \exp \left(-\frac{\nu_{\epsilon}(|\boldsymbol{\zeta}|)}{\mathrm{Kn}}\left(t-\tau_{ \pm}^{b}\right)\right) .
\end{aligned}
$$

Here, the term $f\left(x_{i}-\zeta_{i}\left(t-\tau_{ \pm}^{b}\right), \zeta_{i}, \tau_{ \pm}^{b}\right)$ is expressed more explicitly as

$$
f\left(x_{i}-\zeta_{i}\left(t-\tau_{ \pm}^{b}\right), \zeta_{i}, \tau_{ \pm}^{b}\right)= \begin{cases}f_{w \pm}\left(x_{2}^{b}, x_{3}^{b}, \zeta_{i}, t^{b}\right), & \text { for } \zeta_{i} \in \Gamma_{ \pm}, \\ f_{0}, & \text { for } \zeta_{i} \notin \Gamma_{ \pm} .\end{cases}
$$


If we substitute Eq. (28) into Eq. (18b), then we have

$$
\begin{aligned}
& \mp \frac{\rho_{w \pm}\left(x_{2}, x_{3}, t\right)}{2 \sqrt{\pi}}=\int_{\Omega_{\mp}} c_{1} f_{0} d \boldsymbol{\zeta}+ \int_{\Gamma_{ \pm} \cap \Omega_{\mp}} c_{1} f_{0} \exp \left(-\frac{\nu_{\epsilon}(|\boldsymbol{\zeta}|)}{\mathrm{Kn}}\left(t-t^{b}\right)\right) \\
& \times\left[\rho_{w \pm}^{b} \exp \left(\zeta_{1}^{2}-\left[\zeta_{1}-v_{w}^{b}\right]^{2}\right)-1\right] d \boldsymbol{\zeta} \\
& \text { for } \quad x_{i} \in \partial S_{ \pm}(t),
\end{aligned}
$$

where

$$
\rho_{w \pm}^{b}=\rho_{w \pm}\left(x_{2}^{b}, x_{3}^{b}, t^{b}\right), \quad v_{w}^{b}=v_{w}\left(t^{b}\right) .
$$

Equation (30) yields the expression of $\rho_{w \pm}\left(x_{2}, x_{3}, t\right)$ in terms of $\rho_{w \pm}, x_{w}$, and $v_{w}$ in the past. It could be interpreted as the integral equation for $\rho_{w \pm}$ if we suppose that $x_{w}$ and $v_{w}$ are known. Equation (30) is the key equation for our time-marching solution of the original system.

\subsection{Axisymmetry}

In the three-dimensional case $(d=3)$, we assume that the problem is axisymmetric with respect to the $x_{1}$ axis and reduce the number of the independent variables. Let us introduce the cylindrical coordinate system $\left(x_{1}, r, \theta\right)$ for $x_{i}$ as

$$
x_{1}=x_{1}, \quad x_{2}=r \cos \theta, \quad x_{3}=r \sin \theta,
$$

with $r \in[0, \infty)$ and $\theta \in[0,2 \pi)$, and assume that $\rho_{w \pm}$ is independent of $\theta$ in the cylindrical coordinate system, i.e.,

$$
\tilde{\rho}_{w \pm}(r, t)=\rho_{w \pm}\left(x_{2}, x_{3}, t\right) .
$$

We further introduce some additional notations:

$$
\begin{aligned}
\partial \tilde{S}_{ \pm}(t) & =\left\{\left(x_{1}, r, \theta\right) \in \mathbb{R} \times \mathbb{R}_{+} \times[0,2 \pi) \mid x_{1}=x_{w}(t) \pm 0, r<L / 2\right\}, \\
\Gamma_{ \pm}^{\perp} & =\left\{\zeta_{1} \in \mathbb{R} \mid{ }^{\exists} t^{b}>0,\left(x_{1}, r, \theta\right) \in \partial \tilde{S}_{ \pm}(t)\right\}, \\
\Omega_{ \pm}^{\perp} & =\left\{\zeta_{1} \in \mathbb{R} \mid \zeta_{1} \gtrless v_{w}(t)\right\} .
\end{aligned}
$$

If we express $x_{2}^{b}$ and $x_{3}^{b}$ as

$$
x_{2}^{b}=r^{b} \cos \left(\theta+\theta^{b}\right), \quad x_{3}^{b}=r^{b} \sin \left(\theta+\theta^{b}\right),
$$

using the polar coordinates in the $x_{2} x_{3}$ plane, then Eq. (25) is recast as

$$
\begin{aligned}
& r^{b} \cos \left(\theta+\theta^{b}\right)=r \cos \theta-\zeta_{2}\left(t-t^{b}\right), \\
& r^{b} \sin \left(\theta+\theta^{b}\right)=r \sin \theta-\zeta_{3}\left(t-t^{b}\right) .
\end{aligned}
$$

Let us transform the integration variables from $\left(\zeta_{1}, \zeta_{2}, \zeta_{3}\right)$ to $\left(\zeta_{1}, r^{b}, \theta^{b}\right)$ in Eq. (30) by the use of Eq. (36), noting that $t^{b}=t^{b}\left(\zeta_{1}, t\right)$. Then, we have

$$
\mp \frac{\tilde{\rho}_{w \pm}(r, t)}{2 \sqrt{\pi}}=H_{1 \pm}(t)+P_{1 \pm}\left[\tilde{\rho}_{w \pm}\right](r, t), \quad \text { for } \quad\left(x_{1}, r, \theta\right) \in \partial \tilde{S}_{ \pm},
$$


where $H_{1 \pm}(t)$ and $P_{1 \pm}\left[\tilde{\rho}_{w \pm}\right](r, t)$ are the function and the operator, which are defined in a more general form in the following.

The function $H_{n \pm}(t)$ is defined by

$$
H_{n \pm}(t)=\int_{\Omega_{\mp}} c_{1}^{n} f_{0} d \boldsymbol{\zeta}
$$

or, more explicitly,

$$
\begin{aligned}
& H_{1 \pm}(t)=\mp \frac{1}{2 \sqrt{\pi}} \exp \left(-v_{w}^{2}(t)\right)-\frac{1}{2} v_{w}(t) \operatorname{erfc}\left(\mp v_{w}(t)\right), \\
& H_{2 \pm}(t)= \pm \frac{v_{w}(t)}{2 \sqrt{\pi}} \exp \left(-v_{w}^{2}(t)\right)+\frac{1}{2}\left[v_{w}(t)+\frac{1}{2}\right] \operatorname{erfc}\left(\mp v_{w}(t)\right),
\end{aligned}
$$

where $\operatorname{erfc}(x)$ is the complementary error function:

$$
\operatorname{erfc}(x)=\frac{2}{\sqrt{\pi}} \int_{x}^{\infty} \exp \left(-y^{2}\right) d y=1-\operatorname{erf}(x) .
$$

The operator $P_{n \pm}\left[\tilde{\rho}_{w \pm}\right](r, t)$ is defined as

$$
P_{n \pm}\left[\tilde{\rho}_{w \pm}\right](r, t)=\int_{\Omega_{\ddagger}^{\perp} \cap \Gamma_{ \pm}^{\perp}} c_{1}^{n} F\left[\tilde{\rho}_{w \pm}\right]\left(r, \zeta_{1}, t ; t^{b}\right) d \zeta_{1},
$$

where

$$
\begin{gathered}
F\left[\tilde{\rho}_{w \pm}\right]\left(r, \zeta_{1}, t ; t^{b}\right)=\int_{0}^{L / 2} K\left(\left|\zeta_{1}\right|, r^{b}, r, t-t^{b} ; \mathrm{Kn}, \epsilon\right) \\
\times\left[\tilde{\rho}_{w \pm}\left(r^{b}, t^{b}\right) \exp \left(\zeta_{1}^{2}-\left[\zeta_{1}-v_{w}^{b}\right]^{2}\right)-1\right] d r^{b} \\
K\left(\left|\zeta_{1}\right|, r^{b}, r, t-t^{b} ; \mathrm{Kn}, \epsilon\right) \\
=\frac{2 r^{b}}{\pi^{3 / 2}\left(t-t^{b}\right)^{2}} \int_{0}^{\pi} \exp \left(-\zeta^{2}\left(\theta^{b}\right)-\frac{\nu_{\epsilon}\left(\zeta\left(\theta^{b}\right)\right)}{\mathrm{Kn}}\left(t-t^{b}\right)\right) d \theta^{b} \\
\zeta\left(\theta^{b}\right)=\sqrt{\zeta_{1}^{2}+\frac{\left(r-r^{b}\right)^{2}+2 r^{b} r\left(1-\cos \theta^{b}\right)}{\left(t-t^{b}\right)^{2}}}
\end{gathered}
$$

With these notations, the average drag acting on the disk per unit area (22) is recast as

$$
\begin{array}{r} 
\pm G_{ \pm}(t)=\frac{8}{L^{2}} \int_{0}^{L / 2}\left[H_{2 \pm}(t)+P_{2 \pm}\left[\tilde{\rho}_{w \pm}\right](r, t)+\frac{1}{4} \tilde{\rho}_{w \pm}(r, t)\right] r d r \\
\text { for }\left(x_{1}, r, \theta\right) \in \partial \tilde{S}_{ \pm} .
\end{array}
$$


For a collisionless gas [Eq. (4)], which is given by the limit as $\mathrm{Kn} \rightarrow \infty$ in the special Lorentz gas [Eq. (12)], we have the analytic expression of $K$ [Eq. (41b)], i.e.,

$$
\begin{aligned}
K_{\infty} & \equiv K\left(\left|\zeta_{1}\right|, r^{b}, r, t-t^{b} ; \infty, \epsilon\right) \\
& =\frac{2 r^{b}}{\left(t-t^{b}\right)^{2} \sqrt{\pi}} \exp \left(-\zeta_{1}^{2}-\left(\frac{r-r^{b}}{t-t^{b}}\right)^{2}\right) \bar{I}_{0}\left(\frac{2 r^{b} r}{\left(t-t^{b}\right)^{2}}\right),
\end{aligned}
$$

where $\bar{I}_{0}(x)$ is defined by

$$
\begin{aligned}
& \bar{I}_{0}(x)=\exp (-|x|) I_{0}(x), \\
& I_{0}(x)=\frac{1}{\pi} \int_{0}^{\pi} \exp (x \cos \theta) d \theta .
\end{aligned}
$$

Here, $I_{0}(x)$ is the modified Bessel function of the first kind of order zero.

\section{Numerical Analysis}

In this section, we explain the numerical solution method for the three-dimensional (axisymmetric) case.

\subsection{Numerical scheme for equation of motion}

We first consider the equation of motion of the disk (19). Let us introduce the following discrete variables:

$$
t^{(n)}=n \Delta t, \quad x_{w}^{(n)}=x_{w}\left(t^{(n)}\right), \quad v_{w}^{(n)}=v_{w}\left(t^{(n)}\right), \quad G^{(n)}=G\left[f\left(\cdot, t^{(n)}\right)\right],
$$

where $\Delta t$ is a constant (time interval). Suppose that all the quantities have been obtained up to $t=t^{(n)}$. Then, we compute the position $x_{w}^{(n+1)}$ and velocity $v_{w}^{(n+1)}$ of the disk as well as the $\operatorname{drag} G^{(n+1)}$ at $t=t^{(n+1)}$ by the predictor-corrector method explained below.

For brevity, we introduce the notation

$$
\begin{aligned}
& \mathbf{X}=\left[\begin{array}{l}
x_{w} \\
v_{w}
\end{array}\right], \quad \mathrm{F}=\left[\begin{array}{c}
v_{w} \\
-x_{w}-M^{-1} G[f]
\end{array}\right], \\
& \mathbf{X}^{(n)}=\left[\begin{array}{c}
x_{w}^{(n)} \\
v_{w}^{(n)}
\end{array}\right], \quad \mathrm{F}^{(n)}=\left[\begin{array}{c}
v_{w}^{(n)} \\
-x_{w}^{(n)}-M^{-1} G^{(n)}
\end{array}\right],
\end{aligned}
$$

and write the equation of motion (19) as

$$
\frac{d \mathrm{X}}{d t}=\mathrm{F},
$$


which leads to

$$
\mathrm{X}^{(n+1)}=\mathrm{X}^{(n)}+\int_{t^{(n)}}^{t^{(n+1)}} \mathrm{F} d t .
$$

We first obtain the predicted value $\widehat{\mathrm{X}}^{(n+1)}$ of $\mathrm{X}^{(n+1)}$ by approximating $\mathrm{F}$ in Eq. (48) by the $l_{P}$-degree polynomial of $t$ based on the values $\mathrm{F}^{(n)}, \mathrm{F}^{(n-1)}, \ldots, \mathrm{F}^{\left(n-l_{P}\right)}$, i.e.,

$$
\widehat{\mathrm{X}}^{(n+1)}=\mathrm{X}^{(n)}+\Delta t \sum_{l=0}^{l_{P}} w_{P}^{(n-l)} \mathrm{F}^{(n-l)},
$$

where the weights $w_{P}^{(n)}, \ldots, w_{P}^{\left(n-l_{P}\right)}$ are determined according to the polynomial approximation mentioned above. We set $l_{P}=3$ in this paper $\left(l_{p}=n\right.$ for $\left.n \leq 2\right)$. With this $\widehat{X}^{(n+1)}$, we compute the predicted value $\widehat{F}^{(n+1)}$ (or $\widehat{G}^{(n+1)}$ ) by the procedure that will be described in Sec. 5.2. Then, we compute $X^{(n+1)}$ by approximating $\mathrm{F}$ in Eq. (48) by the $l_{C}$-degree polynomial of $t$ based on the values $\widehat{\mathrm{F}}^{(n+1)}$, $\mathrm{F}^{(n)}, \ldots, \mathrm{F}^{\left(n+1-l_{C}\right)}$, i.e.,

$$
\mathrm{X}^{(n+1)}=\mathrm{X}^{(n)}+\Delta t\left(w_{C}^{(n+1)} \widehat{\mathrm{F}}^{(n+1)}+\sum_{l=0}^{l_{C}-1} w_{C}^{(n-l)} \mathrm{F}^{(n-l)}\right),
$$

where the weights $w_{C}^{(n+1)}, \ldots, w_{C}^{\left(n+1-l_{C}\right)}$ are determined similarly. We set $l_{C}=4$ in the present paper $\left(l_{C}=n\right.$ for $\left.n \leq 3\right)$. Finally, with the new value $\mathrm{X}^{(n+1)}$, we compute $G^{(n+1)}$ (see Sec. 5.2) to prepare for the next step.

Once $x_{w}^{(n+1)}$ and $v_{w}^{(n+1)}$ are obtained, we approximate the trajectory $x_{w}(t)$ in the time interval $t \in\left[t^{(n)}, t^{(n+1)}\right]$ by the cubic polynomial $\psi_{n}(t)$ determined by the following conditions:

$$
\begin{aligned}
& \psi_{n}\left(t^{(n)}\right)=x_{w}^{(n)}, \quad \psi_{n}\left(t^{(n+1)}\right)=x_{w}^{(n+1)}, \\
& \left.\frac{d \psi_{n}}{d t}\right|_{t=t^{(n)}}=v_{w}^{(n)},\left.\quad \frac{d \psi_{n}}{d t}\right|_{t=t^{(n+1)}}=v_{w}^{(n+1)} .
\end{aligned}
$$

The approximated trajectory $x_{w}(t)=\psi_{n}(t)$ in the interval $t \in\left[t^{(n)}, t^{(n+1)}\right](n=$ $0,1,2, \ldots)$ will be used in Sec. 5.3.

\subsection{Numerical scheme for computation of the drag force}

In this subsection, we describe the method of computation of the drag force $G^{(n+1)}$ at $t=t^{(n+1)}$, supposing that the quantities at previous time steps $\left(t=t^{(k)}, k=\right.$ $0,1, \ldots, n)$ are all known and that $x_{w}^{(n+1)}$ and $v_{w}^{(n+1)}$ are also known [cf. Sec. 5.1]. We first introduce grid points in the $r$ and $\theta$ coordinates in the cylindrical coordinate system (32), i.e.,

$$
\begin{aligned}
& r^{(i)}=(L / 2)\left(i / N_{r}\right), \quad\left(i=0, \ldots, N_{r}\right), \\
& \theta^{(j)}=\pi\left(j / N_{\theta}\right), \quad\left(j=0, \ldots, N_{\theta}\right),
\end{aligned}
$$


and denote

$$
\tilde{\rho}_{w \pm}^{(i, k)}=\tilde{\rho}_{w \pm}\left(r^{(i)}, t^{(k)}\right) .
$$

In addition, we choose the grid points $\zeta_{1}^{(k)}$ for $\zeta_{1}$ in such a way that $t^{b}\left(\zeta_{1}^{(k)}, t^{(n+1)}\right)$ [Eq. (24)] falls on the discrete time $t^{(k)}$.

The first step is to obtain $\tilde{\rho}_{w \pm}^{(i, n+1)}$ by the use of Eq. (37) at $r=r^{(i)}$ and $t=t^{(n+1)}$. Since $H_{1 \pm}\left(t^{(n+1)}\right)$ can be obtained from the explicit expression (38b), our major concern is to compute $P_{1 \pm}\left[\tilde{\rho}_{w \pm}\right]\left(r^{(i)}, t^{(n+1)}\right)$ [Eq. (40)], for which we need to compute $F\left[\tilde{\rho}_{w \pm}\right]\left(r^{(i)}, \zeta_{1}^{(k)}, t^{(n+1)} ; t^{b}\left(\zeta_{1}^{(k)}, t^{(n+1)}\right)\right)$. This can be computed for $k=0,1, \ldots, n$ from Eq. (41) because $\rho_{w \pm}^{(i, k)}$ and $v_{w}\left(t^{(k)}\right)$ are known for $i=0, \ldots, N_{r}$ and $k=0, \ldots, n$ [Note that $v_{w}^{b}=v_{w}\left(t^{b}\left(\zeta_{1}^{(k)}, t^{(n+1)}\right)\right)$ in Eq. (41)]. The integration with respect to $\theta^{b}$ in Eq. (41b) and that with respect to $r^{b}$ in Eq. (41a) are carried out by the Simpson rule. Then $P_{1 \pm}\left[\tilde{\rho}_{w \pm}\right]\left(r^{(i)}, t^{(n+1)}\right)$ is computed from Eq. (40), where the integration with respect to $\zeta_{1}$ is performed by the trapezoidal rule. In this integration, $F\left[\tilde{\rho}_{w \pm}\right]\left(r^{(i)}, \zeta_{1}^{(k)}, t^{(n+1)} ; t^{b}\left(\zeta_{1}^{(k)}, t^{(n+1)}\right)\right)$ at $k=n+1$, which corresponds to the end point of the range of integration with respect to $\zeta_{1}$, is not required, since the integrand vanishes there because of the factor $c_{1}$. It should be noted that $t^{b}\left(\zeta_{1}, t^{(n+1)}\right)$, as a function of $\zeta_{1}$, exhibits discontinuities depending on the shape of the trajectory in the past, $x_{w}(s)$ for $s \in[0, t)$. Therefore, for accurate numerical integration, we need the precise locations of the discontinuities as well as the values of the integrand at both sides of the discontinuities. The detailed description how to handle the discontinuities will be given in the next subsection.

Once we obtain $\tilde{\rho}_{w \pm}^{(i, n+1)}$ by the procedure mentioned above, we can compute $G_{ \pm}\left(t^{(n+1)}\right)$ from Eq. (42). Here, $H_{2 \pm}\left(t^{n+1)}\right)$ is obtained from Eq. (38c), and $P_{2 \pm}\left[\tilde{\rho}_{w \pm}\right]\left(r^{(i)}, t^{(n+1)}\right)$ is computed from Eq. (40), in the same way as $P_{1 \pm}\left[\tilde{\rho}_{w \pm}\right]$ $\left(r^{(i)}, t^{(n+1)}\right)$ in the preceding paragraph. Then, the integration with respect to $r$ in Eq. (42) is carried out by the Simpson rule. Then, the average drag $G^{(n+1)}$ at $t=t^{(n+1)}$ is obtained as the sum of $G_{+}\left(t^{(n+1)}\right)$ and $G_{-}\left(t^{(n+1)}\right)$ [Eq. (21)].

The predicted value $\widehat{G}^{(n+1)}$ of $G^{(n+1)}$, which appeared in Sec. 5.1, can be obtained in the same way as $G^{(n+1)}$, using the predicted values $\widehat{\mathrm{X}}^{(n+1)}$ (i.e., the predicted values of $x_{w}^{(n+1)}$ and $v_{w}^{(n+1)}$ ) instead of $x_{w}^{(n+1)}$ and $v_{w}^{(n+1)}$.

\subsection{Numerical scheme for computation of discontinuity}

As we mentioned in Sec. 5.2, $t^{b}\left(\zeta_{1}, t^{(n+1)}\right)$ may be a piecewise continuous function of $\zeta_{1}$ with (a finite number of) discontinuities. In this subsection, we will give a brief sketch how to obtain the positions of discontinuities and the values of $t^{b}$ at both sides of the discontinuities. For simplicity, we discuss only the "+" side, i.e., for $x_{i} \in \partial \tilde{S}_{+}\left(t^{(n+1)}\right)$. The situation is shown in Fig. 2, i.e., the actual trajectory $x_{w}(t)\left[0 \leq t \leq t^{(n+1)}=25\right]$ is shown for $d=1, M=4, L=1, x_{w 0}=1$, $v_{w 0}=0$, and $\mathrm{Kn}=\infty$ (collisionless gas) in Fig. 2(b), and the corresponding $t^{b}$ at $t=25$ is shown as the function of $\zeta_{1}$ in Fig. 2(a). Then, it is obvious that the positions of the discontinuities and the values of $t^{b}$ there are obtained by inspecting the tangential lines of the trajectory. To be more specific, we first solve the 


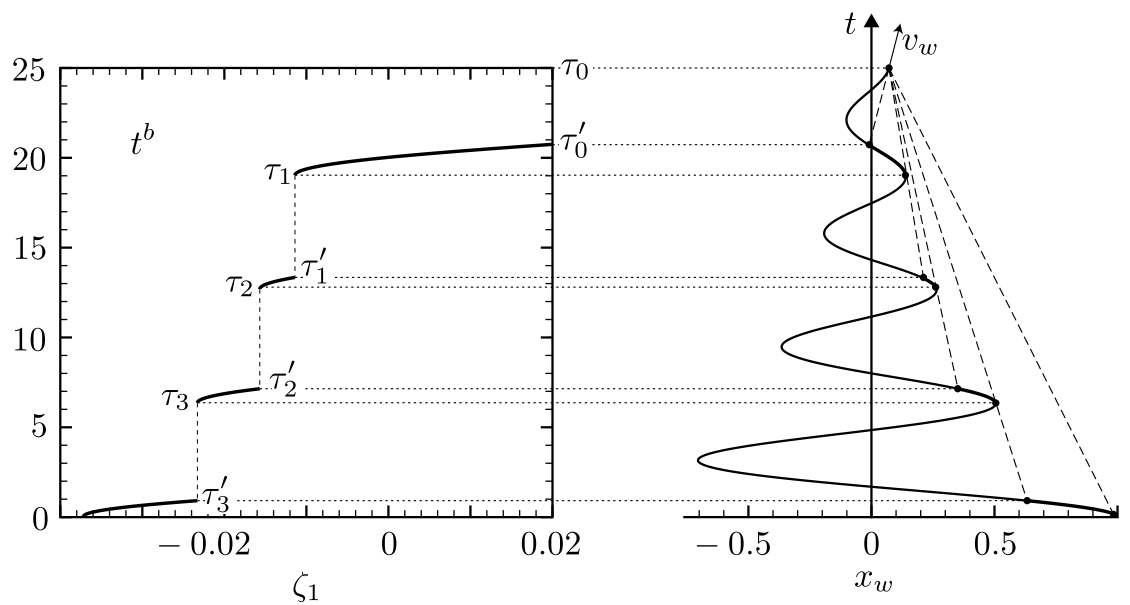

(a)

(b)

Fig. 2 The discontinuities of the function $t^{b}\left(\zeta_{1}, t\right)$ at a given $t$. (a) The $t^{b}\left(\zeta_{1}, t\right)$ at $t=25$ as the function of $\zeta_{1}$. (b) The trajectory $x_{w}(t)$ of the disk for $0 \leq t \leq 25$. The parameters are set as $d=3, M=4, L=1, x_{w 0}=1, v_{w 0}=0$, and $\mathrm{Kn}=\infty$ (collisionless gas).

following equation to obtain $\tau$ :

$$
x_{w}^{(n+1)}-\left.t^{(n+1)} \frac{d \psi_{k}}{d t}\right|_{t=\tau}=\psi_{k}(\tau)-\left.\tau \frac{d \psi_{k}}{d t}\right|_{t=\tau}, \quad \text { for } \quad k=0, \ldots, n,
$$

recalling that the trajectory $x_{w}(t)$ for $t \in\left[0, t^{(n+1)}\right]$ has been approximated piecewisely by cubic polynomials $\psi_{k}(k=0, \ldots, n)$ [cf. the last paragraph of Sec. 5.1]. If $\tau$ satisfies the condition for $t^{b}$ [Eq. (24) with $\left.t=t^{(n+1)}\right]$ and $\tau \in\left[t^{(k)}, t^{(k+1)}\right]$, then $t^{b}$ is discontinuous at $\zeta_{1}=\eta$ such that $t^{b}\left(\eta, t^{(n+1)}\right)=\tau$. By definition, $\tau$ is the value of $t^{b}\left(\zeta_{1}, t^{(n+1)}\right)$ at $\zeta_{1}=\eta+0$. If we denote by $\tau^{\prime}$ the value of $t^{b}\left(\zeta_{1}, t^{(n+1)}\right)$ at $\zeta_{1}=\eta-0$, then it is obtained as

$$
\begin{aligned}
& \tau^{\prime}=\max \left\{\psi_{k}(\tau)-\left.\tau \frac{d \psi_{k}}{d t}\right|_{t=\tau}=\psi_{k^{\prime}}\left(\tau^{\prime}\right)-\left.\tau^{\prime} \frac{d \psi_{k}}{d t}\right|_{t=\tau}\right\}, \\
&\left(\tau \in\left[t^{(k)}, t^{(k+1)}\right], \quad \tau^{\prime} \in\left[t^{\left(k^{\prime}\right)}, t^{\left(k^{\prime}+1\right)}\right], \quad k \geq k^{\prime}\right) .
\end{aligned}
$$

If there is no $k^{\prime}$ satisfying Eq. (56), then $\tau^{\prime}=0$. In Fig. 2(a), $\tau$ 's and $\tau^{\prime \prime}$ s are numbered as $t^{(n+1)}=\tau_{0}>\tau_{0}^{\prime}>\tau_{1}>\tau_{1}^{\prime}>\tau_{2}>\tau_{2}^{\prime}>\cdots$. Equations (55) and (56) are solved by the Newton method.

\subsection{Data for computation}

The numerical results that will be shown in Sec. 6 are based on $\Delta t=0.02$ and $N_{r}=16$ and $N_{\theta}=32$ for $d=3$. We have confirmed, with computations using different grid systems, that this sytem is enough for necessary accuracy. The 


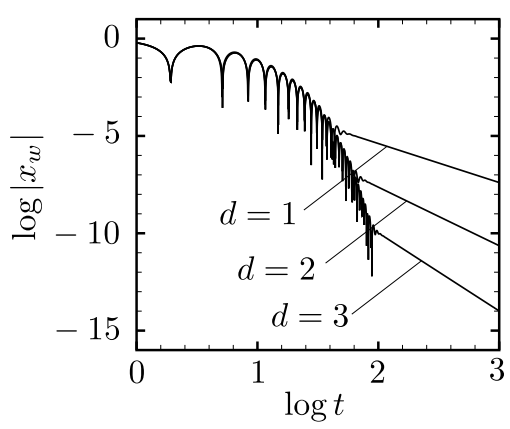

(a)

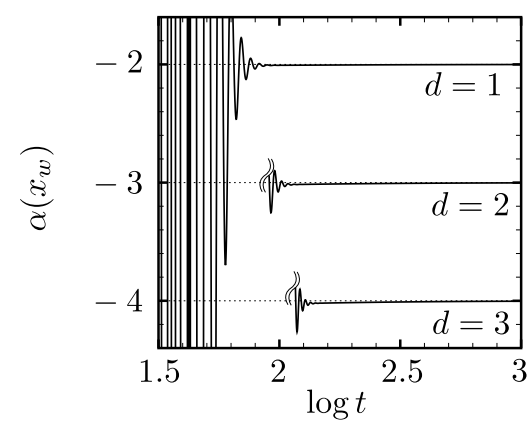

(b)

Fig. 3 The decay of the displacement $x_{w}(t)$ for $d=1,2$, and 3 in the case of $M=4, L=1$ $\left(d=2\right.$ and 3 ), $x_{w 0}=1$, and $v_{w 0}=0$ (collisionless gas: $\left.K n=\infty\right)$. (a) $\log _{10}\left|x_{w}\right|$ vs $\log _{10} t$. (b) The gradient $\alpha\left(x_{w}\right)$ of the curve in panel (a) vs $\log _{10} t$.

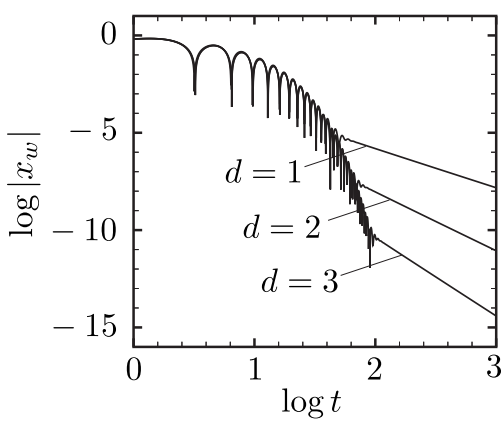

(a)

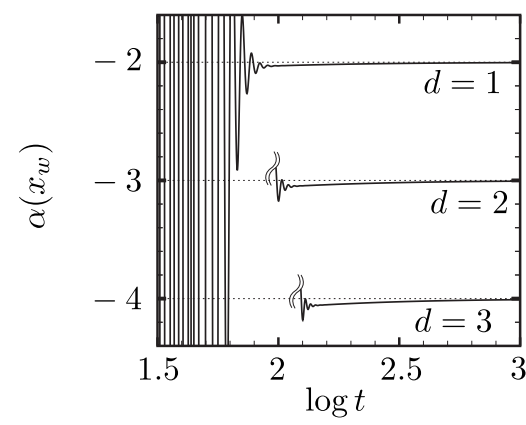

(b)

Fig. 4 The decay of the displacement $x_{w}(t)$ for $d=1,2$, and 3 in the case of $M=4, L=1$ $\left(d=2\right.$ and 3 ), $x_{w 0}=0$, and $v_{w 0}=1$ (collisionless gas: $\mathrm{Kn}=\infty$ ). (a) $\log _{10}\left|x_{w}\right|$ vs $\log _{10} t$. (b) The gradient $\alpha\left(x_{w}\right)$ of the curve in panel (a) vs $\log _{10} t$.

computation was carried out with quadruple precision. We have used fast algorithms for the error and Bessel functions provided by T. Ooura, available from his home page (http://www.kurims.kyoto-u.ac.jp/ ooura/index.html). The algorithms are for double precision, but we have confirmed that they give an accuracy of 19 significant figures if they are used in a quadruple-precision computation.

The computation has been carried out on a PC cluster with CPU: Intel(R) Core 2 Extreme QX9650 3.0GHz(4CPU) $\times 8$.

\section{Results}

In this section, we present some numerical results, focusing our attention on the manner of decay of the displacement $x_{w}(t)$ of the disk at long times. 
Table 1 The values of the gradient $\alpha\left(x_{w}\right)$ of the curve $\log _{10}\left|x_{w}\right|$ vs $\log _{10} t$ for $d=1,2$, and 3 and for $M=1,4$, and 10 in the case of $L=1(d=2$ and 3$), x_{w 0}=1$, and $v_{w 0}=0$ (collisionless gas: $\mathrm{Kn}=\infty$ ). See Fig. 3 for $M=4$.

\begin{tabular}{|c|c|c|c|c|c|c|c|c|c|}
\hline \multirow[b]{3}{*}{$t \quad \log t$} & \multicolumn{9}{|c|}{$-\alpha\left(x_{w}\right)$} \\
\hline & \multicolumn{3}{|c|}{$d=1$} & \multicolumn{3}{|c|}{$d=2$} & \multicolumn{3}{|c|}{$d=3$} \\
\hline & $M=10$ & $M=4$ & $M=1$ & $M=10$ & $M=4$ & $M=1$ & $M=10$ & $M=4$ & $M=1$ \\
\hline $10.00 \quad 1.0$ & - $^{*}$ & - $^{*}$ & 3.6368 & - $^{*}$ & - $^{*}$ & 7.5391 & - $^{*}$ & - $^{*}$ & 8.5635 \\
\hline 31.621 .5 & - $^{*}$ & - $^{*}$ & 2.1620 & - $^{*}$ & - $^{*}$ & 3.3034 & — $^{*}$ & - $^{*}$ & 4.4575 \\
\hline $100.00 \quad 2.0$ & - $^{*}$ & 2.0074 & 2.0463 & - $^{*}$ & 3.0476 & 3.0813 & - $^{*}$ & - $^{*}$ & 4.1167 \\
\hline $316.23 \quad 2.5$ & 1.9821 & 2.0029 & 2.0143 & 2.9941 & 3.0066 & 3.0246 & - $^{*}$ & 4.0100 & 4.0351 \\
\hline $398.11 \quad 2.6$ & 860 & 2.0024 & 2.0113 & 2.9863 & 3.0053 & 3.0195 & 3.9896 & 4.0081 & 4.0277 \\
\hline $\begin{array}{ll}630.96 & 2.8\end{array}$ & 914 & 2.0015 & 2.0071 & 2.9916 & 3.0034 & 3.0122 & 3.9916 & 4.0052 & 4.0174 \\
\hline $1000.00 \quad 3.0$ & 1.9946 & 2.0010 & 2.0045 & 2.9948 & 3.0022 & 3.0077 & 3.9952 & 4.0036 & 4.0113 \\
\hline $1995.26 \quad 3.3$ & 1.9974 & $\cdots$ & 2.0022 & 2.9974 & $\cdots$ & 3.0038 & $\cdots$ & $\cdots$ & $\cdots$ \\
\hline $3162.28 \quad 3.5$ & 1.9983 & $\cdots$ & 2.0014 & 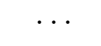 & $\cdots$ & $\cdots$ & $\cdots$ & $\cdots$ & $\cdots$ \\
\hline $10000.00 \quad 4.0$ & 1.9995 & $\cdots$ & 2.0004 & & $\cdots$ & $\cdots$ & $\ldots$ & $\cdots$ & $\cdots$ \\
\hline
\end{tabular}

* Values are omitted because of the strong oscillation of $\alpha\left(x_{w}\right)$ [see e.g. Fig. 3(b)].

Table 2 The values of the gradient $\alpha\left(x_{w}\right)$ of the curve $\log _{10}\left|x_{w}\right|$ vs $\log _{10} t$ for $d=1,2$, and 3 in the case of $M=4, L=1(d=2$ and 3$), x_{w 0}=0$, and $v_{w 0}=1$ (collisionless gas: $\mathrm{Kn}=\infty)$. See Fig. 4 .

\begin{tabular}{rr||ccc}
\hline \multicolumn{1}{c||}{$t$} & \multicolumn{3}{c}{$-\alpha\left(x_{w}\right)$} \\
\cline { 3 - 5 }$t$ & $\log t$ & $d=1$ & $d=2$ & $d=3$ \\
\hline 100.00 & 2.0 & 2.0309 & 3.1730 & $-^{*}$ \\
158.49 & 2.2 & 2.0197 & 3.0364 & 4.0521 \\
251.19 & 2.4 & 2.0127 & 3.0233 & 4.0333 \\
398.11 & 2.6 & 2.0081 & 3.0148 & 4.0212 \\
630.96 & 2.8 & 2.0052 & 3.0094 & 4.0135 \\
1000.00 & 3.0 & 2.0033 & 3.0059 & 4.0092 \\
\hline
\end{tabular}

${ }^{*}$ Values are omitted because of the strong oscillation of $\alpha\left(x_{w}\right)$ [see e.g. Fig. 4(b)].

\subsection{Collisionless gas}

First, we show the results for the collisionless gas (cf. Sec. 2.2.1), which can also be regarded as the special case $\mathrm{Kn}=\infty$ of Eq. (12). Figure 3 contains the decay properties of the displacement $x_{w}(t)$ of the disk at long times for the $d$-dimensional problems ( $d=1,2$, and 3; see Fig. 1$)$ in the case of $M=4, L=1$ ( $d=2$ and 3 ), $x_{w 0}=1$, and $v_{w 0}=0$ : Fig. 3(a) shows the plot $\log _{10}\left|x_{w}(t)\right|$ versus $\log _{10} t$, and Fig. 3(b) shows the gradient $\alpha\left(x_{w}\right)$ of the curve in Fig. 3(a), i.e.,

$$
\alpha\left(x_{w}\right)=d \log _{10}\left|x_{w}(t)\right| / d \log _{10} t .
$$

Table 1 shows the values of $\alpha\left(x_{w}\right)$ corresponding to Fig. 3(b) as well as its values for $M=1$ and 10. Figure 4 is the counterpart of Fig. 3 with different initial conditions of the disk, $x_{w 0}=0$ and $v_{w 0}=1$, and Table 2 shows the numerical values of $\alpha\left(x_{w}\right)$ corresponding to Fig. 4(b). These figures and tables supplement our earlier work [6], in which only the case of $d=1$ is investigated.

The results contained in Figs. 3 and 4 and Tables 1 and 2, together with those of other cases that are not shown in this paper, provide some pieces of numerical 


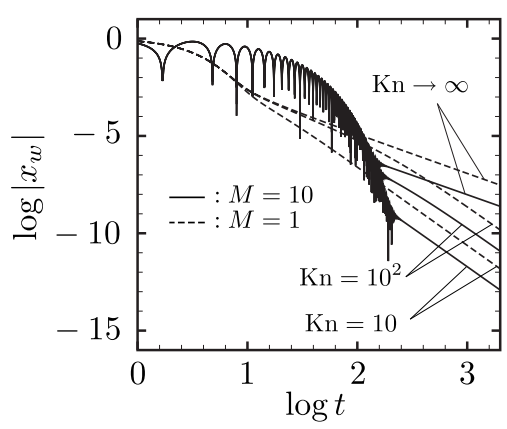

(a)

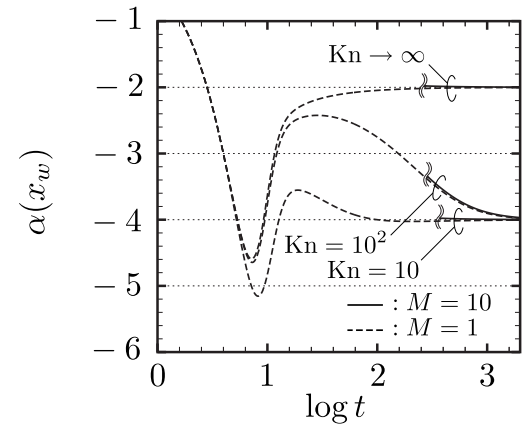

(b)

Fig. 5 The decay of the displacement $x_{w}(t)$ for $d=1, x_{w 0}=1$, and $v_{w 0}=0$ [special Lorentz gas with resting obstacle: Eq. (12) with $\epsilon=0$ ]. (a) $\log _{10}\left|x_{w}\right|$ vs $\log _{10} t$. (b) The gradient $\alpha\left(x_{w}\right)$ of the curve in panel (a) vs $\log _{10} t$. The solid line is for $M=10$ (oscillatory decay), and the dashed line is for $M=1$ (monotonic decay). The results for $\mathrm{Kn}=10,10^{2}$, and $\infty$ (collisionless gas) are shown in the figures.

evidence for the decay rate (2), i.e.,

$$
\left|x_{w}(t)\right| \approx C / t^{d+1},
$$

for large $t$, where and hereafter $C$ symbolically denotes a constant with respect to $t$ that may depend on other parameters.

As was proven mathematically in [1-4] and then confirmed numerically in [5, 6], the slow algebraic decay is attributed to the long-memory effect caused by multiple collisions of a gas molecule with the disk. In general, when a body is moving freely in a collisionless gas under the action of an external force, the body is accelerated or decelerated by the force. In this situation, the molecules that are reflected by the body at early times may hit the body again and again at later times. Such molecules transfer information about the body at early stages directly to the body at later times and may affect the motion of the body long time later. That is, they give rise to a long-memory effect. In fact, if the effect of the multiple collisions is neglected, the decay is exponential in time [1,2]. Our next interest is how the decay rate is modified if the long-memory effect is destroyed by some means. This is the reason why we consider the toy model Eq. (12) based on the special Lorentz gas.

\subsection{Special Lorentz gas: Case of resting obstacles}

We first consider Eq. (12) with $\epsilon=0$, which corresponds to the case where the obstacles are at rest in the special Lorentz gas. We show some results in this case in Figs. 5 and 6 as well as Tables 3 and 4. Figure 5(a) shows the curves $\log _{10}\left|x_{w}(t)\right|$ versus $\log _{10} t$ in the one-dimensional problem $(d=1)$ with initial condition $x_{w 0}=$ 1 and $v_{w 0}=0$ for $M=1$ (monotonic decay) and 10 (oscillatory decay) and for $\mathrm{Kn}=10$ and $10^{2}$ together with the collisionless case $(\mathrm{Kn}=\infty)$, and the gradient $\alpha\left(x_{w}\right)$ of each curve is plotted versus $\log _{10} t$ in Fig. 5(b). Some values of $\alpha\left(x_{w}\right)$ 




(a)

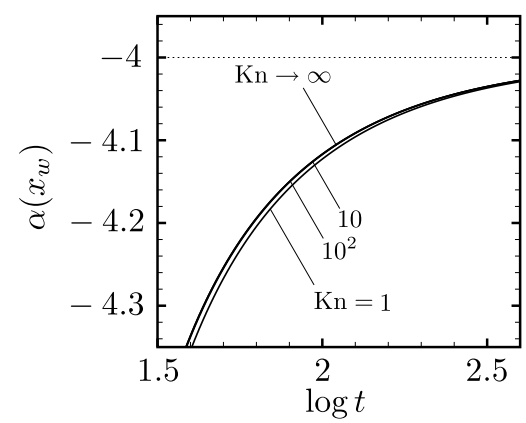

(b)

Fig. 6 The decay of the displacement $x_{w}(t)$ for $d=3, M=1, L=1, x_{w 0}=1$, and $v_{w 0}=0$ [special Lorentz gas with resting obstacle: Eq. (12) with $\epsilon=0$ ]. (a) $\log _{10}\left|x_{w}\right|$ vs $\log _{10} t$. (b) The gradient $\alpha\left(x_{w}\right)$ of the curve in panel (a) vs $\log _{10} t$. The results for $\mathrm{Kn}=1,10,10^{2}$, and $\infty$ (collisionless gas) are shown in the figures.

Table 3 The values of the gradient $\alpha\left(x_{w}\right)$ of the curve $\log _{10}\left|x_{w}\right|$ vs $\log _{10} t$ for $d=1, x_{w 0}=$ 1 , and $v_{w 0}=0$ [special Lorentz gas with resting obstacle: Eq. (12) with $\epsilon=0$ ]. See Fig. 5.

\begin{tabular}{|c|c|c|c|c|c|c|c|}
\hline & \multicolumn{6}{|c|}{$-\alpha\left(x_{w}\right)$} \\
\hline & & \multicolumn{2}{|c|}{$\mathrm{Kn} \rightarrow \infty$} & \multicolumn{2}{|c|}{$\mathrm{Kn}=10^{2}$} & \multicolumn{2}{|c|}{$\mathrm{Kn}=10$} \\
\hline$t$ & $\log t$ & $M=10$ & $M=1$ & $M=10$ & $M=1$ & $M=10$ & $M=1$ \\
\hline 316.23 & 2.5 & 1.9821 & 2.0143 & 3.4116 & 3.4517 & 3.9827 & 4.0229 \\
\hline 1000.00 & 3.0 & 1.9946 & 2.0045 & 3.8891 & 3.9041 & 3.9938 & 4.0097 \\
\hline 1995.26 & 3.3 & 1.9974 & 2.0022 & 3.9684 & 3.9762 & 3.9972 & 4.0052 \\
\hline 10000.00 & 4.0 & 1.9995 & 2.0004 & $\ldots$ & $\ldots$ & $\ldots$ & $\ldots$ \\
\hline
\end{tabular}

Table 4 The values of the gradient $\alpha\left(x_{w}\right)$ of the curve $\log _{10}\left|x_{w}\right| \operatorname{vs} \log _{10} t$ for $d=3, M=1$, $L=1, x_{w 0}=1$, and $v_{w 0}=0$ [special Lorentz gas with resting obstacle: Eq. (12) with $\epsilon=0$ ]. See Fig. 6.

\begin{tabular}{|c|c|c|c|c|c|}
\hline \multirow[b]{2}{*}{$t$} & \multirow[b]{2}{*}{$\log t$} & \multicolumn{4}{|c|}{$-\alpha\left(x_{w}\right)$} \\
\hline & & $\mathrm{Kn} \rightarrow \infty$ & $\mathrm{Kn}=10^{2}$ & $\mathrm{Kn}=10$ & $\mathrm{Kn}=1$ \\
\hline 10.00 & 1.0 & 8.5635 & 8.5641 & 8.5698 & 8.6089 \\
\hline 25.12 & 1.4 & 4.8025 & 4.8041 & 4.8192 & 5.0253 \\
\hline 63.10 & 1.8 & 4.1942 & 4.1943 & 4.1952 & 4.2043 \\
\hline 158.49 & 2.2 & 4.0716 & 4.0716 & 4.0719 & 4.0752 \\
\hline 398.11 & 2.6 & 4.0277 & 4.0277 & 4.0279 & 4.0291 \\
\hline
\end{tabular}

at large times are shown in Table 3. Figure 6 is the corresponding figure in the three-dimensional problem $(d=3)$ with initial condition $x_{w 0}=1$ and $v_{w 0}=0$ for $M=1$ and $L=1$ and for $\mathrm{Kn}=1,10,10^{2}$ and $\infty$ (collisionless gas). Table 4 shows some values of the gradient $\alpha\left(x_{w}\right)$ in Fig. 6 at large times.

From these results, it is likely that the displacement $x_{w}(t)$ of the disk decays as

$$
\left|x_{w}(t)\right| \approx C / t^{4}
$$




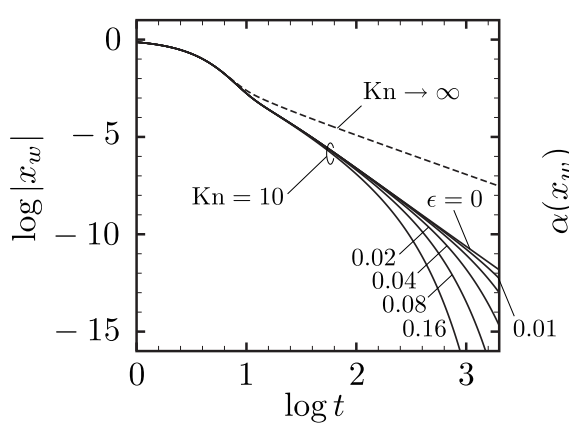

(a)

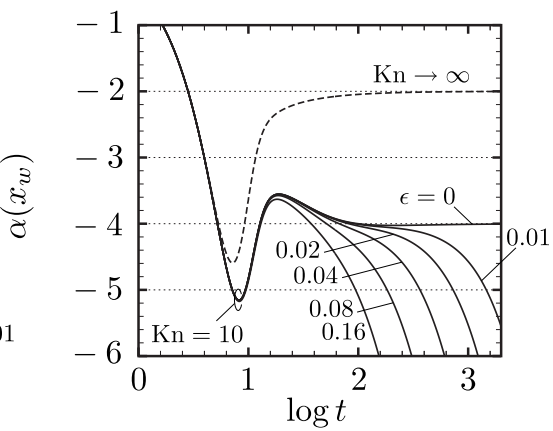

(b)

Fig. 7 The decay of the displacement $x_{w}(t)$ for $d=1, M=1, x_{w 0}=1$, and $v_{w 0}=0$ [special Lorentz gas with moving obstacle: Eq. (12)]. (a) $\log _{10}\left|x_{w}\right|$ vs $\log _{10} t$. (b) The gradient $\alpha\left(x_{w}\right)$ of the curve in panel (a) vs $\log _{10} t$.

for large $t$, independent of the dimension $d$ of the problem. This is different from Eq. (58) for a collisionless gas, but the rate is still algebraic. Unlike the collisionless case, the gas molecules reflected by the disk generally hit the obstacles before hitting the disk again. Therefore, the long-memory effect in the collisionless gas is destroyed in the present case. However, since the obstacles are at rest, slow molecules among the reflected molecules may not interact with the obstacles before they are hit again by the disk. In this sense, the long-memory effect is not completely destroyed. This may be the reason why we still have an algebraic decay. In the present setting, the obstacles are three dimensional (spheres of uniform size) and randomly distributed in the three-dimensional space, irrespective of the dimension $d$ of the problem shown in Fig. 1. Therefore, it is natural that the decay rate (59) is independent of $d$. For instance, the rate may change if the obstacles are two-dimensional cylinders distributed randomly in the $x_{1} x_{2}$ space in the two dimensional problem $(d=2)$.

\subsection{Special Lorentz gas: Case of moving obstacles}

Finally we consider Eq. (12) with non-zero $\epsilon$, which corresponds to the case where the obstacles are moving with low speed (cf. Appendix). Some results for the decay property of the displacement are shown in Figs. 7-9 as well as Tables 5-7. Figure 7(a) shows the curves $\log _{10}\left|x_{w}(t)\right|$ versus $\log _{10} t$ in the one-dimensional problem $(d=1)$ with initial condition $x_{w 0}=1$ and $v_{w 0}=0$ for $M=1$ (monotonic decay), $\mathrm{Kn}=10$ and $\infty$ (collisionless case), and various values of $\epsilon$ including $\epsilon=0$ (case of resting obstacles). The gradient $\alpha\left(x_{w}\right)$ of each curve in Fig. 7(a) is plotted versus $\log _{10} t$ in Fig. 7(b), and some values of $\alpha\left(x_{w}\right)$ at large times are shown in Table 5. Figure 8 and Table 6 are the corresponding figure and table for oscillatory decay, that is, for $d=1, M=10, \mathrm{Kn}=10$ and $\infty$, and various $\epsilon$ with initial condition $x_{w 0}=1$ and $v_{w 0}=0$. Then, Fig. 9 and Table 7 show the corresponding results in the three-dimensional problem $(d=3)$ for $M=1$ (monotonic 


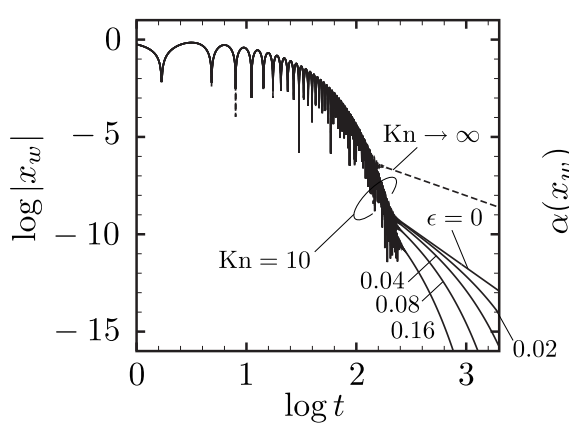

(a)

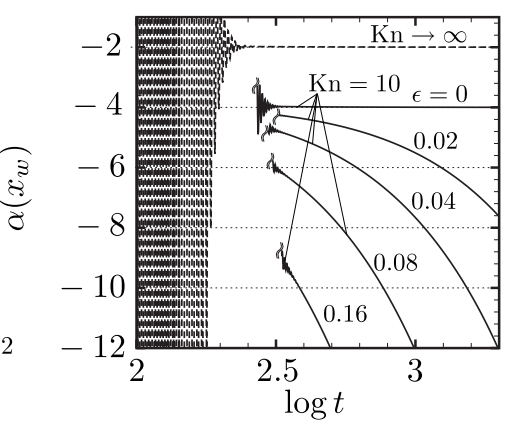

(b)

Fig. 8 The decay of the displacement $x_{w}(t)$ for $d=1, M=10, x_{w 0}=1$, and $v_{w 0}=0$ [special Lorentz gas with moving obstacle: Eq. (12)]. (a) $\log _{10}\left|x_{w}\right|$ vs $\log _{10} t$. (b) The gradient $\alpha\left(x_{w}\right)$ of the curve in panel (a) vs $\log _{10} t$.

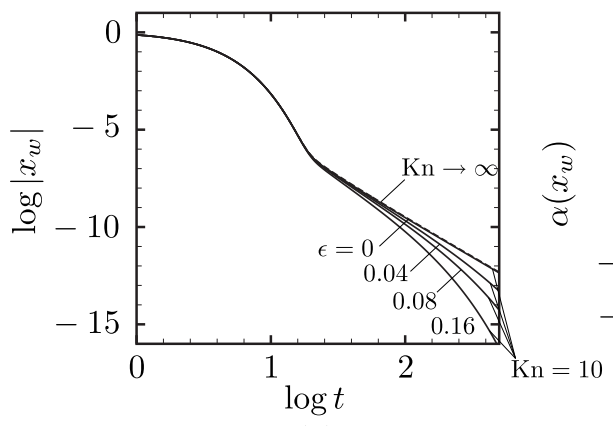

(a)

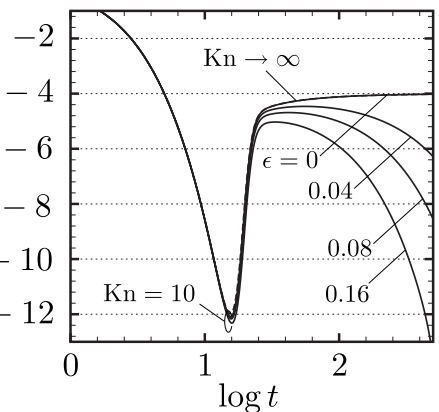

(b)

Fig. 9 The decay of the displacement $x_{w}(t)$ for $d=3, M=1, L=1, x_{w 0}=1$, and $v_{w 0}=0$ [special Lorentz gas with moving obstacle: Eq. (12)]. (a) $\log _{10}\left|x_{w}\right|$ vs $\log _{10} t$. (b) The gradient



decay), $L=1, \mathrm{Kn}=10$ and $\infty$, and various $\epsilon$ with initial condition $x_{w 0}=1$ and $v_{w 0}=0$.

It is seen from these results that, when $\epsilon \neq 0$, the gradient $\alpha\left(x_{w}\right)$ decreases faster and faster as time $t$ increases for large $t$. This suggests that the decay rate is exponential in $t$. To see it more clearly, we plot, in Fig. $10(\mathrm{a}), \log _{10}\left|t^{4} x_{w}(t)\right|$ versus $t$ in the same case as in Fig. 9 and, in Fig. 10(b), the gradient of the curve in Fig. 10(a), i.e.,

$$
\beta\left(x_{w}\right)=d \log _{10}\left|t^{4} x_{w}(t)\right| / d t .
$$

Figure 10(b) indicates that the gradient $\beta\left(x_{w}\right)$ tends to approach a non-zero constant value, depending on $\epsilon$, when $\epsilon \neq 0$. For $\epsilon=0.16$, the curve deviates from the constant value for $t$ larger than about 400 . This may be due to the numerical error, since $\left|x_{w}(t)\right|$ is too small for accurate computation in this case [see Fig. 9(a)]. Some values of $\beta\left(x_{w}\right)$ are shown for large $t$ in Table 8. From these results, the 




(a)

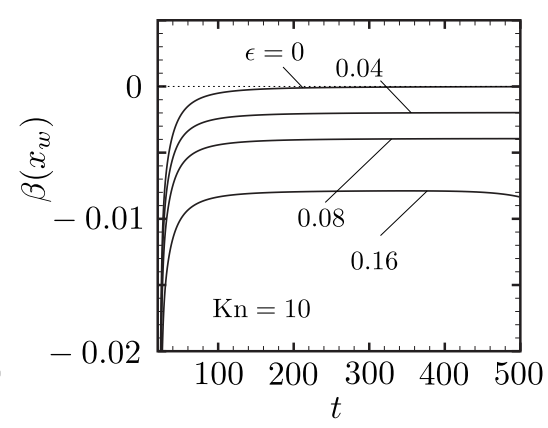

(b)

Fig. 10 The decay of $t^{4} x_{w}(t)$ in the case of Fig. 9 and Table 7. (a) $\log _{10}\left|t^{4} x_{w}\right|$ vs $t$. (b) The gradient $\beta\left(x_{w}\right)$ of the curve in panel (a) vs $t$.

decay of $x_{w}(t)$ for large $t$ is likely to be

$$
\left|x_{w}(t)\right| \approx C t^{-4} \exp (-\bar{\beta} t)
$$

with constant $\bar{\beta}$ (depending on the parameters) for $d=3$. However, for $d=1$ and 2 , the numerical result does not show clear decay property as in the case of $d=3$.

Contrary to the case of resting obstacles, even slow molecules among the reflected molecules are hit by the moving obstacles in the present case of $\epsilon \neq 0$. Therefore, almost no information about the motion of the disk is transmitted directly to the later time. In other words, the long-memory effect caused by multiple collisions of a gas molecule with the disk is eliminated. As the consequence, we

Table 5 The values of the gradient $\alpha\left(x_{w}\right)$ of the curve $\log _{10}\left|x_{w}\right|$ vs $\log _{10} t$ for $d=1, M=1$, $x_{w 0}=1$, and $v_{w 0}=0$ [special Lorentz gas with moving obstacle: Eq. (12)]. See Fig. 7.

\begin{tabular}{|c|c|c|c|c|c|c|c|c|}
\hline \multirow[b]{3}{*}{$t$} & \multirow[b]{3}{*}{$\log t$} & \multicolumn{7}{|c|}{$-\alpha\left(x_{w}\right)$} \\
\hline & & \multirow{2}{*}{$\begin{array}{c}\mathrm{Kn} \rightarrow \infty \\
-\end{array}$} & \multicolumn{6}{|c|}{$\mathrm{Kn}=10$} \\
\hline & & & $\epsilon=0$ & $\epsilon=0.01$ & $\epsilon=0.02$ & $\epsilon=0.04$ & $\epsilon=0.08$ & $\epsilon=0.16$ \\
\hline 316.23 & 2.5 & 2.0143 & 4.0229 & 4.1044 & 4.2963 & 4.8191 & 6.0808 & 8.8256 \\
\hline 630.96 & 2.8 & 2.0071 & 4.0143 & 4.2839 & 4.8181 & 6.0871 & 8.8150 & 14.4522 \\
\hline 1000.00 & 3.0 & 2.0045 & 4.0097 & 4.5708 & 5.5291 & 7.6797 & 12.1092 & $\ldots$ \\
\hline 1995.26 & 3.3 & 2.0022 & 4.0052 & 5.5526 & 7.6319 & 12.0896 & $\ldots$ & $\ldots$ \\
\hline
\end{tabular}

Table 6 The values of the gradient $\alpha\left(x_{w}\right)$ of the curve $\log _{10}\left|x_{w}\right|$ vs $\log _{10} t$ for $d=1, M=$ $10, x_{w 0}=1$, and $v_{w 0}=0$ [special Lorentz gas with moving obstacle: Eq. (12)]. See Fig. 8.

\begin{tabular}{rr||c|rrrrr}
\hline \multicolumn{1}{c||}{} & \multicolumn{6}{|c}{$-\alpha\left(x_{w}\right)$} \\
\cline { 3 - 8 } & & $\mathrm{Kn} \rightarrow \infty$ & \multicolumn{5}{c}{$\mathrm{Kn}=10$} \\
\cline { 3 - 8 }$t$ & $\log t$ & - & $\epsilon=0$ & $\epsilon=0.02$ & $\epsilon=0.04$ & $\epsilon=0.08$ & $\epsilon=0.16$ \\
\hline 316.23 & 2.5 & 1.9821 & 3.9827 & 4.2596 & 4.7898 & 6.0830 & 9.2442 \\
630.96 & 2.8 & 1.9914 & 3.9888 & 4.7939 & 6.0642 & 8.7931 & 14.4310 \\
1000.00 & 3.0 & 1.9946 & 3.9938 & 5.5144 & 7.6655 & 12.0959 & $\ldots$ \\
1995.26 & 3.3 & 1.9974 & 3.9972 & 7.6254 & 12.0866 & $\ldots$ & $\ldots$ \\
\hline
\end{tabular}


Table 7 The values of the gradient $\alpha\left(x_{w}\right)$ of the curve $\log _{10}\left|x_{w}\right|$ vs $\log _{10} t$ for $d=3, M=1$, $L=1, x_{w 0}=1$, and $v_{w 0}=0$ [special Lorentz gas with moving obstacle: Eq. (12)]. See Fig. 9 .

\begin{tabular}{cr||c|cccr}
\hline \multicolumn{1}{l||}{} & \multicolumn{5}{|c}{$-\alpha\left(x_{w}\right)$} \\
\cline { 3 - 7 } & \multicolumn{1}{c||}{$\mathrm{Kn} \rightarrow \infty$} & \multicolumn{4}{|c}{$\mathrm{Kn}=10$} \\
\cline { 3 - 7 }$t$ & $\log t$ & - & $\epsilon=0$ & $\epsilon=0.04$ & $\epsilon=0.08$ & $\epsilon=0.16$ \\
\hline 100.00 & 2.0 & 4.1167 & 4.1173 & 4.5613 & 5.0168 & 5.9224 \\
158.49 & 2.2 & 4.0716 & 4.0719 & 4.7827 & 5.5006 & 6.9332 \\
251.19 & 2.4 & 4.0444 & 4.0446 & 5.1755 & 6.3109 & 8.5797 \\
398.11 & 2.6 & 4.0277 & 4.0279 & 5.8230 & 7.6214 & 11.2453 \\
\hline
\end{tabular}

Table 8 The values of the gradient $\beta\left(x_{w}\right)$ of the curve $\log _{10}\left|t^{4} x_{w}\right|$ vs $t$ in the case of Fig. 9 and Table 7.

\begin{tabular}{|c|c|c|c|c|c|}
\hline \multirow[b]{2}{*}{$t$} & \multirow[b]{2}{*}{$\log t$} & \multicolumn{4}{|c|}{$-\beta\left(x_{w}\right)$} \\
\hline & & $\epsilon=0$ & $\epsilon=0.04$ & $\epsilon=0.08$ & $\epsilon=0.16$ \\
\hline 100.00 & 2.000 & $5.0957 \times 10$ & $2.4379 \times 10^{-3}$ & $4.4162 \times 10^{-3}$ & $8.3495 \times 10^{-}$ \\
\hline 149.97 & 2.176 & $2075 \times 10^{-4}$ & $2.1668 \times 10^{-3}$ & $4.1350 \times 10^{-3}$ & $8.0610 \times 10^{-}$ \\
\hline 199.99 & 2.301 & $1.2260 \times 10^{-4}$ & $2.0749 \times 10^{-3}$ & $4.0396 \times 10^{-3}$ & $7.9631 \times 10^{-}$ \\
\hline 250.03 & 2.398 & $7.7865 \times 10^{-5}$ & $2.0330 \times 10^{-3}$ & $3.9961 \times 10^{-3}$ & 7.918 \\
\hline 299 & 2.477 & $5.3861 \times 10^{-5}$ & $2.0106 \times 10^{-3}$ & $3.9728 \times 10^{-3}$ & $7.8965 \times 10^{-}$ \\
\hline 34 & 2.544 & $3.9427 \times 10^{-5}$ & $1.9971 \times 10^{-3}$ & $3.9590 \times 10^{-3}$ & - $^{*}$ \\
\hline 399.94 & 2.602 & 3.0113 & $1.9884 \times 10^{-3}$ & $3.9503 \times 10^{-3}$ & - $^{*}$ \\
\hline 449 & 2.653 & $2.3771 \times 10^{-5}$ & $1.9825 \times 10^{-3}$ & $3.9450 \times 10^{-3}$ & —* \\
\hline 498.88 & 2.698 & $1.9299 \times 10^{-5}$ & $1.9785 \times 10^{-3}$ & $3.9426 \times 10^{-3}$ & — $^{*}$ \\
\hline
\end{tabular}

* Values are omitted because of the loss of accuracy [see Fig. 10(b)].

have a faster decay, such as given by Eq. (61). The decay is faster for larger $\epsilon$ because the obstacles moving with higher speed have more chances to collide with the gas molecules.

\section{Concluding remarks}

In the present study, we have investigated unsteady motion of a disk in a gas under the action of an external force obeying Hooke's law, with special interest in the manner of decay of the motion caused by the drag force exerted by the gas. We first considered the case where the gas is a highly rarefied collisionless gas (freemolecular gas or the Knudsen gas) and provided some pieces of numerical evidence for the decay of the displacement described by Eq. (2) or (58). These results complement our previous numerical results for the one-dimensional case $(d=1)$ [6] as well as the rigorous mathematical results for the case of non-oscillatory decay [2] [specular reflection rather than diffuse reflection is employed in [2], so that the decay rate is given by Eq. (1)]. The slow algebraic decay is attributed to the long-memory effect caused by multiple collisions of a gas molecules with the disk, as proven mathematically in [1-4] and confirmed numerically in [5,6]. In fact, the decay becomes exponentially fast if the multiple collisions are neglected by assuming that all the molecules incident on the disk come from the initial velocity distribution. 
Our next interest was to see how the decay rate is modified when the longmemory effect is destroyed by some means. For this purpose, we have introduced a toy model, Eq. (12), whose physical basis is a type of the Lorentz gas consisting of free-streaming gas (vapor) molecules and randomly distributed spherical obstacles (droplets of the liquid or solid phase of the vapor) with which the gas molecules interact. Our numerical results show the following: The decay rate is still algebraic when $\epsilon=0$ in Eq. (12), which corresponds to the case of resting obstacles, but we have a faster decay rate that is likely to be exponential in time when $\epsilon \neq 0$, which corresponds to the case of moving obstacles. This result seems to be natural, since the moving obstacles destroy the memory of the gas molecules almost completely. However, if we consider a real gas with collisions between gas molecules, the manner of decay of the motion of the disk may be different from the case of the present toy model. In this connection, it should be mentioned that the decay rate of translational and rotational motion (monotonic and oscillatory decay) of a spherical body was investigated mathematically when the surrounding fluid is the viscous Stokes fluid $[8,9]$. In this case, the decay rate was proven to be algebraic in time. This fact also provides a motivation to study the present problem for the gas with intermolecular collisions. This will be a subject of the next stage of our study.

Finally, it should be remarked that the present problem has some similarity to the so-called piston problem, which is a fundamental problem in statistical physics (see [14-16] and the references therein). In [17,18], for instance, an unsteady motion of a piston has been investigated numerically, using particle methods, when the movable piston is placed in a closed container, the gas is collisionless, and the boundary condition is specular reflection. In this case, the oscillating motion of the piston is caused by instability of a mechanical equilibrium [19], rather than an external force as considered in the present paper.

\section{A Special Lorentz gas: Derivation of Eqs. (5) and (12)}

In this section, we consider a physical model that can be said to be a special version of the Lorentz gas $[10,11]$ and give a physical derivation of the corresponding equations, Eqs. (5) and (12), along the same line as the original derivation of the Boltzmann equation [12]. In particular, we follow the argument in [13].

\section{A.1 Physical model}

We consider, as in the main text, a gas composed of hard-sphere molecules of mass $m$ and radius $r$, whose velocity distribution function is denoted by $f_{*}\left(X_{i}, \xi_{i}, t_{*}\right)$. In the gas, spherical obstacles of uniform size (mass $m_{s}$ and radius $r_{s}$ ) are distributed randomly. We suppose that the gas is the vapor of a substance and the obstacles are made of the condensed phase of the same substance, so that evaporation and condensation of the gas may take place on the surface of the obstacles. A physical image may be given by a system composed of a dilute water vapor and tiny water droplets suspended in it, though only a monatomic gas is considered in the present paper. As described in Sec. 2.2.2, the obstacles are moving with velocity $\xi_{s i}$. Our assumptions on the model are as follows:

1. Gas molecules hitting an obstacle are absorbed in it.

2. The obstacles emit the gas molecules according to a given velocity distribution $f_{* v}\left(\xi_{i}-\xi_{s i}\right)$, which is uniform in space and constant in time. 
3. The motion of the obstacles are not affected by the interaction with the gas molecules or other obstacles, and their velocities $\xi_{s i}$ are distributed according to a given velocity distribution $f_{* s}\left(\xi_{s i}\right)$, which is uniform in space and constant in time.

4. Collisions between gas molecules can be neglected.

5. No external force acts on the gas molecules.

The first and second assumptions correspond to the usual boundary condition for the Boltzmann equation on the interface on which evaporation or condensation is taking place (for instance, the complete condensation condition [7]). The fourth assumption is realized when the number density of the gas molecules $n_{*}$ and that of the obstacles $n_{* s}$, defined by

$$
n_{*}=\frac{1}{m} \int_{\mathbb{R}^{3}} f_{*} d \boldsymbol{\xi}, \quad n_{* s}=\frac{1}{m_{s}} \int_{\mathbb{R}^{3}} f_{* s} d \boldsymbol{\xi}_{s},
$$

satisfy the condition

$$
\max _{X_{i} \in \mathbb{R}^{3}, t \in \mathbb{R}_{+}} n_{*} r^{2} \ll n_{* s} r_{s}^{2},
$$

as will be discussed in Sec. A.2.2.

\section{A.2 Kinetic equations for the model}

We first derive the general form of the kinetic equation for the physical model introduced in Sec. A.1 (Sec. A.2.1). Then we simplify the kinetic equation with the help of special choices of $f_{* s}$ and $f_{* v}$ (Sec. A.2.3). In the mean time, the discussion about the mean free path will be given in Sec. A.2.2.

\section{A.2.1 General Form}

Proposition 1 For the physical model described in Sec. A.l (the special Lorentz gas), the velocity distribution function of the gas molecules $f_{*}\left(X_{i}, \xi_{i}, t_{*}\right)$ satisfies Eqs. (5) and (6).

Proof Let $d \boldsymbol{X} d \boldsymbol{\xi}$ denote a small neighborhood of $\left(X_{i}, \xi_{i}\right)$ in the six-dimensional phase space. Then, $\left(f_{*} / m\right) d \boldsymbol{X} d \boldsymbol{\xi}$ represents the number of gas molecules in $d \boldsymbol{X} d \boldsymbol{\xi}$. Suppose that the volume $d \boldsymbol{X} d \boldsymbol{\xi}$ is moving according to the equation of motion:

$$
\frac{d X_{i}}{d t_{*}}=\xi_{i}, \quad \frac{d \xi_{i}}{d t_{*}}=0
$$

Therefore, the volume $d \boldsymbol{X} d \boldsymbol{\xi}$ at time $t_{*}$ moves to the volume $d \overline{\boldsymbol{X}} d \overline{\boldsymbol{\xi}}$ at time $\bar{t}_{*}=t_{*}+d t_{*}$, which is a small neighborhood of the point

$$
\bar{X}_{i}=X_{i}+\xi_{i} d t_{*}, \quad \bar{\xi}_{i}=\xi_{i},
$$

where $d t_{*}$ is a small increment of time. Since $d \boldsymbol{X} d \boldsymbol{\xi}=d \overline{\boldsymbol{X}} d \overline{\boldsymbol{\xi}}$ holds, the difference between the number of molecules in $d \overline{\boldsymbol{X}} d \overline{\boldsymbol{\xi}}$ and that in $d \boldsymbol{X} d \boldsymbol{\xi}$ is given by

$$
\begin{aligned}
& \frac{1}{m} f_{*}\left(\bar{X}_{i}, \bar{\xi}_{i}, \bar{t}_{*}\right) d \overline{\boldsymbol{X}} d \overline{\boldsymbol{\xi}}-\frac{1}{m} f_{*}\left(X_{i}, \xi_{i}, t_{*}\right) d \boldsymbol{X} d \boldsymbol{\xi} \\
= & \frac{1}{m}\left[\frac{\partial f_{*}}{\partial t_{*}}+\xi_{i} \frac{\partial f_{*}}{\partial X_{i}}+O\left(d t_{*}\right)\right] d \boldsymbol{X} d \boldsymbol{\xi} d t_{*} .
\end{aligned}
$$

This is equal to the increase of the number of molecules in the volume $d \boldsymbol{X} d \boldsymbol{\xi}$ minus its decrease during the time interval $d t_{*}$ because of collisions with the obstacles, which can be expressed as

$$
\frac{1}{m} J_{\text {gain }}\left(\bar{X}_{i}, \bar{\xi}_{i}, \bar{t}_{*}\right) d \overline{\boldsymbol{X}} d \overline{\boldsymbol{\xi}} d t_{*}-\frac{1}{m} J_{\text {loss }}\left(X_{i}, \xi_{i}, t_{*}\right) d \boldsymbol{X} d \boldsymbol{\xi} d t_{*} .
$$






(a)

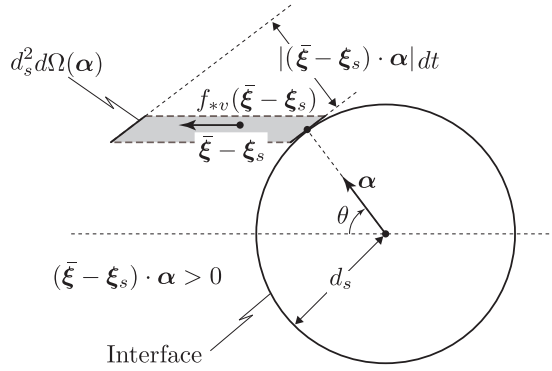

(b)

Fig. 11 Configuration of the collision between gas molecules and an obstacle with velocity $\xi_{s i}$. (a) The loss term. (b) The gain term.

More precisely, the first term in Eq. (67) (the gain term) indicates the number of molecules that are contained in the volume $d \overline{\boldsymbol{X}} d \overline{\boldsymbol{\xi}}$ at time $\bar{t}_{*}$ and have emitted from the obstacles during $d t_{*}$. The second term in Eq. (67) (the loss term) indicates the number of molecules that were contained in the volume $d \boldsymbol{X} d \boldsymbol{\xi}$ at time $t_{*}$ and have hit the obstacles during $d t_{*}$. We recall here that we have neglected the collision between gas molecules (see Sec. A.2.2).

Now we try to derive the explicit form of $J_{\text {loss }}$ and $J_{\text {gain }}$.

Loss Term : $J_{\text {loss }}$ Consider an obstacle moving with a velocity $\boldsymbol{\xi}_{s}$ contained in the volume $d \boldsymbol{X}$. We denote a unit vector at the center of the obstacle by $\boldsymbol{\alpha}$ and the solid-angle element around $\boldsymbol{\alpha}$ by $d \Omega(\boldsymbol{\alpha})$. The number of the molecules with velocity in the small neighborhood $d \boldsymbol{\xi}$ around $\boldsymbol{\xi}$ and hitting the obstacle during $d t_{*}$ is given by the number of the molecules contained in the cylinder with height $\left|\left(\boldsymbol{\xi}-\boldsymbol{\xi}_{s}\right) \cdot \boldsymbol{\alpha}\right| d t_{*}$ and the base area $d_{s}^{2} \Omega(\boldsymbol{\alpha})\left(d_{s}=r+r_{s}\right)$ in Fig. 11(a), i.e.,

$$
\frac{1}{m} f_{*}\left(X_{i}, \xi_{i}, t_{*}\right) d \boldsymbol{\xi} \times\left|\left(\boldsymbol{\xi}-\boldsymbol{\xi}_{s}\right) \cdot \boldsymbol{\alpha}\right| d t_{*} \times d_{s}^{2} d \Omega(\boldsymbol{\alpha}) \times \mathbf{1}_{\left\{\left(\boldsymbol{\xi}-\boldsymbol{\xi}_{s}\right) \cdot \boldsymbol{\alpha}<0\right\}},
$$

where $\mathbf{1}_{A}$ is the characteristic function of a set $A$. By integrating Eq. (68) for all $\boldsymbol{\alpha}$, we obtain the total number of the gas molecules with velocity in $d \boldsymbol{\xi}$ hitting the obstacle during $d t_{*}$, i.e.,

$$
\left(\int_{\text {all } \boldsymbol{\alpha}} \frac{1}{m} f_{*}\left|\left(\boldsymbol{\xi}-\boldsymbol{\xi}_{s}\right) \cdot \boldsymbol{\alpha}\right| \mathbf{1}_{\left\{\left(\boldsymbol{\xi}-\boldsymbol{\xi}_{s}\right) \cdot \boldsymbol{\alpha}<0\right\}} d_{s}^{2} d \Omega(\boldsymbol{\alpha})\right) d \boldsymbol{\xi} d t_{*}=\frac{1}{m} \pi d_{s}^{2}\left|\boldsymbol{\xi}-\boldsymbol{\xi}_{s}\right| f_{*} d \boldsymbol{\xi} d t_{*} .
$$

Since the number of the obstacles contained in the small neighborhood $d \boldsymbol{X} d \boldsymbol{\xi}_{s}$ around $\left(X_{i}, \xi_{s i}\right)$ is given by $\left(1 / m_{s}\right) f_{* s}\left(\boldsymbol{\xi}_{s}\right) d \boldsymbol{X} d \boldsymbol{\xi}_{s}$, the total number of the gas molecules contained in $d \boldsymbol{X} d \boldsymbol{\xi}$ and hitting the obstacles during $d t_{*}$, i.e., $(1 / m) J_{\text {loss }} d \boldsymbol{X} d \boldsymbol{\xi} d t_{*}$, is obtained as

$$
\begin{aligned}
\frac{1}{m} J_{\mathrm{loss}} d \boldsymbol{X} d \boldsymbol{\xi} d t_{*} & =\int_{\xi_{s i} \in \mathbb{R}^{3}}\left(\frac{1}{m} \pi d_{s}^{2}\left|\boldsymbol{\xi}-\boldsymbol{\xi}_{s}\right| f_{*} d \boldsymbol{\xi} d t_{*}\right) \frac{1}{m_{s}} f_{* s}\left(\xi_{s i}\right) d \boldsymbol{X} d \boldsymbol{\xi}_{s} \\
& =\frac{1}{m} \frac{\pi d_{s}^{2}}{m_{s}}\left(\int_{\xi_{s i} \in \mathbb{R}^{3}}\left|\boldsymbol{\xi}-\boldsymbol{\xi}_{s}\right| f_{* s}\left(\xi_{s i}\right) d \boldsymbol{\xi}_{s}\right) f_{*} d \boldsymbol{X} d \boldsymbol{\xi} d t_{*} .
\end{aligned}
$$

Gain Term : $J_{\text {gain }}$ We count the number of gas molecules with velocity in a small neighborhood $d \overline{\boldsymbol{\xi}}$ around $\overline{\boldsymbol{\xi}}$, emitted from an obstacle during $d t_{*}$. Since the distribution function for the emitted vapor molecules is a given function, the same discussion as the loss term holds. The only difference is that $f_{*}$ is replaced by $f_{* v}\left(\bar{\xi}_{i}-\xi_{s i}\right)$ and $\mathbf{1}_{\left\{\left(\boldsymbol{\xi}-\boldsymbol{\xi}_{s}\right) \cdot \boldsymbol{\alpha}<0\right\}}$ by $\mathbf{1}_{\left\{\left(\overline{\boldsymbol{\xi}}-\boldsymbol{\xi}_{s}\right) \cdot \boldsymbol{\alpha}>0\right\}}$ 
in Eq. (68) [see Fig. 11(b)]. Noting that $\bar{\xi}_{i}=\xi_{i}$ [Eq. (65)] and $d \overline{\boldsymbol{X}} d \overline{\boldsymbol{\xi}}=d \boldsymbol{X} d \boldsymbol{\xi}$, we obtain

$$
\begin{aligned}
\frac{1}{m} J_{\text {gain }} d \overline{\boldsymbol{X}} d \overline{\boldsymbol{\xi}} d t_{*} & =\int_{\xi_{s i} \in \mathbb{R}^{3}}\left(\frac{1}{m} \pi d_{s}^{2}\left|\overline{\boldsymbol{\xi}}-\boldsymbol{\xi}_{s}\right| f_{* v}\left(\bar{\xi}_{i}-\xi_{s i}\right) d \overline{\boldsymbol{\xi}} d t_{*}\right) \frac{1}{m_{s}} f_{* s}\left(\xi_{s}\right) d \overline{\boldsymbol{X}} d \boldsymbol{\xi}_{s} \\
& =\frac{1}{m} \frac{\pi d_{s}^{2}}{m_{s}}\left(\int_{\xi_{s i} \in \mathbb{R}^{3}}\left|\boldsymbol{\xi}-\boldsymbol{\xi}_{s}\right| f_{* s}\left(\xi_{s i}\right) f_{* v}\left(\xi_{i}-\xi_{s i}\right) d \boldsymbol{\xi}_{s}\right) d \boldsymbol{X} d \boldsymbol{\xi} d t_{*} .
\end{aligned}
$$

Equating Eq. (66) and Eq. (67) with Eqs. (70) and (71) and neglecting higher-order terms of $d t_{*}$, we obtain Eqs. (5) and (6). $\square$

\section{A.2.2 Mean free path}

In order to discuss the mean free paths of the gas molecules, we consider the case where the gas is in the equilibrium state at rest at temperature $T_{* 0}$ and density $\rho_{* 0}$ (or molecular number density $n_{* 0}$ ), i.e.,

$$
f_{*}=\frac{m n_{* 0}}{\left(2 \pi R_{*} T_{* 0}\right)^{3 / 2}} \exp \left(-\frac{|\boldsymbol{\xi}|^{2}}{2 R_{*} T_{* 0}}\right) .
$$

Then, the mean free path $l_{*}$ of a gas molecule for the collisions against gas molecules is given by [7]

$$
l_{*}=1 / \sqrt{2} \pi(2 r)^{2} n_{* 0} .
$$

On the other hand, from the consideration in Sec. A.2.1, the mean free path $l_{* s}$ of a gas molecules for the collisions against the obstacles is obtained as follows:

$$
\begin{aligned}
l_{* s} & =\left[\frac{\pi d_{s}^{2}}{m_{s}} \int_{\mathbb{R}^{3}} f_{* s}\left(\xi_{s i}\right) \Phi\left(\frac{\left|\boldsymbol{\xi}_{s}\right|}{\sqrt{2 R_{*} T_{* 0}}}\right) d \boldsymbol{\xi}_{s}\right]^{-1}, \\
\Phi(x) & =\frac{1}{2}\left[\exp \left(-x^{2}\right)+\sqrt{\pi}\left(x+\frac{1}{2 x}\right) \operatorname{erf}(x)\right] .
\end{aligned}
$$

Let us consider the ratio $l_{* s} / l_{*}$ under the condition (63). Then it can be estimated as

$$
\begin{aligned}
\frac{l_{* s}}{l_{*}} & =\sqrt{2} \pi(2 r)^{2} n_{* 0}\left[\frac{\pi d_{s}^{2}}{m_{s}} \int_{\mathbb{R}^{3}} f_{* s}\left(\xi_{s i}\right) \Phi\left(\frac{\left|\boldsymbol{\xi}_{s}\right|}{\sqrt{2 R_{*} T_{* 0}}}\right) d \boldsymbol{\xi}_{s}\right]^{-1} \\
& \leq 4 \sqrt{2} \frac{n_{* 0} r^{2}}{n_{* s}\left(r+r_{s}\right)^{2}} \ll 1,
\end{aligned}
$$

where the first inequality is due to the property $\Phi(x) \geq 1$, and the second one due to Eq. (63). If we choose the characteristic length $\left(\sqrt{2 R_{*} T_{* 0}} / \omega_{*}\right)$ (cf. Sec 3.1) much shorter than $l_{*}$ but comparable to or longer than $l_{* s}$, we can neglect the effect of collisions between gas molecules. This legitimates the assumption 4 in Sec. A.1.

\section{A.2.3 Special choice of $f_{* s}$ and $f_{* v}$}

Finally, we derive the simplified version (12) of the kinetic equation (5) with the help of special choices of $f_{* v}$ and $f_{* s}$ and a further assumption.

Let us choose the following Maxwellians as $f_{* v}$ and $f_{* s}$ :

$$
\begin{aligned}
& f_{* v}\left(\xi_{i}-\xi_{s i}\right)=\frac{m n_{* 0}}{\left(2 \pi R_{*} T_{* 0}\right)^{3 / 2}} \exp \left(-\frac{\left|\boldsymbol{\xi}-\boldsymbol{\xi}_{s}\right|^{2}}{2 R_{*} T_{* 0}}\right), \\
& f_{* s}\left(\xi_{s i}\right)=\frac{m_{s} n_{* s}}{\left(2 \pi \Theta_{s}\right)^{3 / 2}} \exp \left(-\frac{\left|\boldsymbol{\xi}_{s}\right|^{2}}{2 \Theta_{s}}\right)
\end{aligned}
$$


where $\Theta_{s}$ is a positive constant, and introduce the following $\epsilon$ :

$$
\epsilon=\sqrt{\frac{\Theta_{s}}{R_{*} T_{* 0}}}
$$

Then, Eqs. (6a) and (6b) are reduced to

$$
\begin{aligned}
\nu\left(\xi_{i}\right) & =2 \sqrt{\pi} n_{* s}\left(r+r_{s}\right)^{2} \sqrt{2 \Theta_{s}} \phi\left(0,|\boldsymbol{\xi}| / \sqrt{2 \Theta_{s}}\right), \\
f_{* \infty}\left(\xi_{i}\right) & =\frac{\phi\left(\epsilon,|\boldsymbol{\xi}| / \sqrt{2 \Theta_{s}}\right)}{\phi\left(0,|\boldsymbol{\xi}| / \sqrt{2 \Theta_{s}}\right)} \frac{m n_{* 0}}{\left(2 \pi R_{*} T_{* 0}\right)^{3 / 2}} \exp \left(-\frac{\epsilon^{2}}{1+\epsilon^{2}} \frac{|\boldsymbol{\xi}|^{2}}{2 \Theta_{s}}\right),
\end{aligned}
$$

where $\phi(a, x)$ is a dimensionless function defined by

$$
\phi(a, x)=\left.\frac{1}{2}\left(1+a^{2}\right)^{-2}\left[\exp \left(-\bar{x}^{2}\right)+\sqrt{\pi}\left(\bar{x}+\frac{1}{2 \bar{x}}\right) \operatorname{erf}(\bar{x})\right]\right|_{\bar{x}=x / \sqrt{1+a^{2}}} .
$$

By the use of the dimensionless variables in Eq. (7), Eq. (5) is recast as

$$
\begin{aligned}
\frac{\partial f}{\partial t}+\zeta_{i} \frac{\partial f}{\partial X_{i}}=\frac{1}{\mathrm{Kn}_{\epsilon}}\left(\frac{2}{\sqrt{\pi}}\right. & \left.\frac{\epsilon}{\sqrt{1+\epsilon^{2}}}\right) \phi\left(0, \epsilon^{-1}|\boldsymbol{\zeta}|\right) \\
& \times\left[\frac{\phi\left(\epsilon, \epsilon^{-1}|\boldsymbol{\zeta}|\right)}{\phi\left(0, \epsilon^{-1}|\boldsymbol{\zeta}|\right)} \frac{1}{\pi^{3 / 2}} \exp \left(-\frac{|\boldsymbol{\zeta}|^{2}}{1+\epsilon^{2}}\right)-f\right],
\end{aligned}
$$

where $\mathrm{Kn}_{\epsilon}$ is defined as [see Eq. (74a)]

$$
\mathrm{Kn}_{\epsilon}=l_{* s} /\left(\sqrt{2 R_{*} T_{* 0}} / \omega_{*}\right)=\left[\pi n_{* s}\left(r+r_{s}\right)^{2} \sqrt{1+\epsilon^{2}}\right]^{-1}\left(\sqrt{2 R_{*} T_{* 0}} / \omega_{*}\right)^{-1} .
$$

We now assume $\epsilon \ll 1$, which means that the speed of motion of the obstacles is much lower than that of the thermal motion of the gas molecules, and neglect the terms of $O\left(\epsilon^{2}\right)$. Then, we finally obtain the equation that is studied in the main text, i.e.,

$$
\frac{\partial f}{\partial t}+\zeta_{i} \frac{\partial f}{\partial x_{i}}=\frac{\nu_{\epsilon}(|\boldsymbol{\zeta}|)}{\mathrm{Kn}}\left[\frac{1}{\pi^{3 / 2}} \exp \left(-|\boldsymbol{\zeta}|^{2}\right)-f\right],
$$

where

$$
\begin{aligned}
& \nu_{\epsilon}(x)=\frac{2}{\sqrt{\pi}} \epsilon \phi\left(0, \epsilon^{-1} x\right)=\frac{\epsilon}{\sqrt{\pi}}\left[\exp \left(-\frac{x^{2}}{\epsilon^{2}}\right)+\sqrt{\pi}\left(\frac{x}{\epsilon}+\frac{\epsilon}{2 x}\right) \operatorname{erf}\left(\frac{x}{\epsilon}\right)\right], \\
& \mathrm{Kn}=l_{* s}(\epsilon=0) /\left(\sqrt{2 R_{*} T_{* 0}} / \omega_{*}\right)=\left[\pi n_{* s}\left(r+r_{s}\right)^{2}\right]^{-1}\left(\sqrt{2 R_{*} T_{* 0}} / \omega_{*}\right)^{-1} .
\end{aligned}
$$

Acknowledgements The authors express their gratitude to Guido Cavallaro, Carlo Marchioro, and Mario Pulvirenti for valuable discussions. Their thanks are also due to Shigeru Takata for stimulating discussions. K.A. wishes to thank ICERM, Brown University for its hospitality during the preparation of this paper.

\section{References}

1. Caprino, S., Marchioro, C., Pulvirenti, M.: Approach to equilibrium in a microscopic model of friction. Commun. Math. Phys. 264, 167-189 (2006).

2. Caprino, S., Cavallaro, G., Marchioro, C.: On a microscopic model of viscous friction. Math. Models Methods Appl. Sci. 17, 1369-1403 (2007).

3. Cavallaro, G.: On the motion of a convex body interacting with a perfect gas in the meanfield approximation. Rendiconti di Matematica. Ser. VII 27, 123-145 (2007). 
4. Aoki, K., Cavallaro, G., Marchioro, C., Pulvirenti, M.: On the motion of a body in thermal equilibrium immersed in a perfect gas. Math. Model. Num. Anal. 42, 263-275 (2008).

5. Aoki, K., Tsuji, T., Cavallaro, G.: Approach to steady motion of a plate moving in a freemolecular gas under a constant external force. Phys. Rev. E. 80, 016309 (2009).

6. Tsuji, T., Aoki, K.: Decay of an oscillating plate in a free-molecular gas. In: Levin, D. A., Wysong, I. J., Garcia, A. L. (eds.) Rarefied Gas Dynamics. AIP, Melville (2011). pp. 140145.

7. Sone, Y.: Molecular Gas Dynamics: Theory, Techniques, and Applications. Birkäuser, Boston (2007); Supplementary Notes and Errata: Kyoto University Research Information Repository (http://hdl.handle.net/2433/66098).

8. Cavallaro, G., Marchioro, C.: On the approach to equilibrium for a pendulum immersed in a Stokes fluid. Math. Models Methods Appl. Sci. 20, 1999-2019 (2010).

9. Cavallaro, G., Marchioro, C., Tsuji, T.: Approach to equilibrium of a rotating sphere in a Stokes flow. Ann. Univ. Ferrara 57, 211-228 (2011).

10. Gallavotti, G.: Statistical Mechanics: A Short Treatise. Springer, Berlin (1999).

11. Caglioti, E., Golse, F.: On the Boltzmann-Grad limit for the two dimensional periodic Lorentz gas. J. Stat. Phys. 141, 264-317 (2010).

12. Boltzmann, L.: Lectures on Gas Theory. Dover, New York (1995).

13. Takata, S.: Invitation to the kinetic theory. J. Jpn. Soc. Fluid Mech. (Nagare) 27, 387-396 (2008) (in Japanese).

14. Gruber, Ch., Piasecki, J.: Stationary motion of the adiabatic piston. Physica A 268, 412-423 (1999).

15. Chernov, N., Lebowitz, J. L., Sinai, Ya.: Scaling dynamics of a massive piston in a cube filled with ideal gas: Exact results. J. Stat. Phys. 109, 529-548 (2002).

16. Lebowitz, J. L., Piasecki, J., Sinai, Ya.: in Hard Ball Systems and the Lorentz Gas, Encyclopedia of Mathematical Sciences Vol. 101 (Springer, New York, 2000), pp. 217-227.

17. Kestemont, E., Van den Broeck, C., Mansour, M. M.: The "adiabatic" piston: And yet it moves. Europhys. Lett. 49, 143-149 (2000).

18. Chernov, N., Lebowitz, J. L.: Dynamics of a massive piston in an ideal gas: Oscillatory motion and approach to equilibrium. J. Stat. Phys. 109, 507-527 (2002).

19. Caglioti, E., Chernov, N., Lebowitz, J. L.: Stability of solutions of hydrodynamic equations describing the scaling limit of a massive piston in an ideal gas. Nonlinearity 17, 897-923 (2004). 